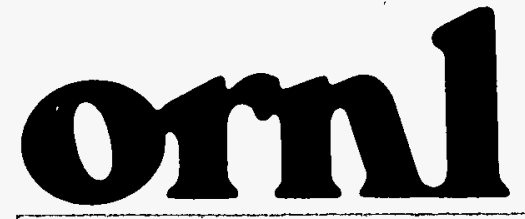

OAK RIDGE NATIONAL LABORATORY

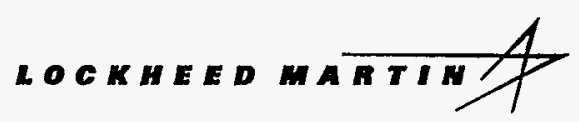

MANAGED AND OPERATED BY LOCKHEED MARTIN ENERGY RESEARCH CORPORATION FOR THE UNTEO STATES DEPARTMEIT OF ENERGY
Results of the Radiological and

Chemical Characterization of Surface Impoundments 3539 and 3540 at Oak Ridge National Laboratory, Oak Ridge, Tennessee

\author{
M. E. Murray \\ D. A. Rose \\ K. S. Brown \\ W. Winton \\ R. A. Dean \\ R. H. Coe III
}

\section{RECEIVED}

MAR 251998

$$
\text { OSTI }
$$

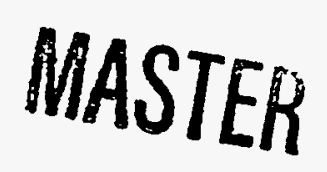

No

DISTRIBUTION OF THIS DOCUAENT IS UNLAMTED 
This report has been reproduced directly from the best available copy.

Available to DOE and DOE contractors from the Office of Scientific and Technical Information, P.O. Box 62, Oak Ridge, TN 37831; prices available from (615) 576-8401, FTS 626-8401.

Available to the public from the National Technical Information Service, U.S. Department of Commerce, 5285 Port Royal Rd., Springfield, VA 22161.

This report was prepared as an account of work sponsored by an agency of the United States Government. Neither the United States Government nor any agency thereof, nor any of their employees, makes any warranty, express or implied, or assumes any legal liability or responsibility for the accuracy, completeness, or usefulness of any information, apparatus, product, or process disclosed, or represents that its use would not infringe privately owned rights. Reference herein to any specific commercial product, process, or service by trade name, trademark, manufacturer, or otherwise, does not necesserily constitute or imply its endorsement, recommendation, or favoring by the United States Government or any agency thereof. The views and opinions of authors expressed herein do not necessarily state or reflect those of the United States Government or any agency thereof. 


\section{DISCLAIMER}

Portions of this document may be illegible electronic image products. Images are produced from the best available original document. 


\title{
Results of the Radiological and Chemical Characterization of Surface Impoundments 3539 and 3540 at Oak Ridge National Laboratory, Oak Ridge, Tennessee
}

\author{
M. E. Murray \\ D. A. Rose \\ K. S. Brown \\ W. Winton \\ R. A. Dean \\ R. H. Coe III
}

Report Issued-March 1998

\author{
Prepared by the \\ Life Sciences Division \\ OAK RIDGE NATIONAL LABORATORY \\ Oak Ridge, Tennessee 37831-6285 \\ managed by \\ LOCKHEED MARTIN ENERGY RESEARCH CORP. \\ for the \\ U.S. DEPARTMENT OF ENERGY \\ under contract DE-AC05-96OR22464
}




\section{CONTENTS}

FIGURES $\ldots \ldots \ldots \ldots \ldots \ldots \ldots \ldots \ldots \ldots \ldots \ldots \ldots \ldots \ldots \ldots \ldots$

TABLES $\ldots \ldots \ldots \ldots \ldots \ldots \ldots \ldots \ldots \ldots \ldots \ldots \ldots \ldots \ldots \ldots \ldots \ldots \ldots$

ACKNOWLEDGMENTS $\ldots \ldots \ldots \ldots \ldots \ldots \ldots \ldots \ldots \ldots \ldots \ldots \ldots \ldots$ ix

EXECUTIVE SUMMARY $\ldots \ldots \ldots \ldots \ldots \ldots \ldots \ldots \ldots \ldots \ldots \ldots \ldots \ldots$

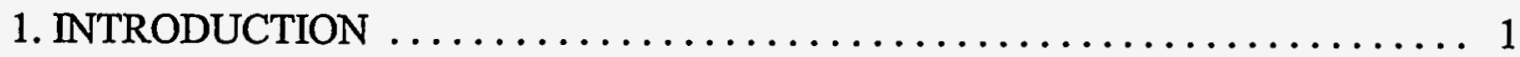

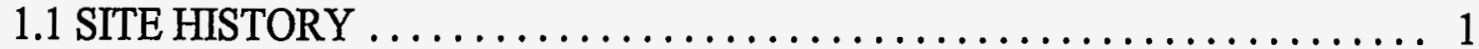

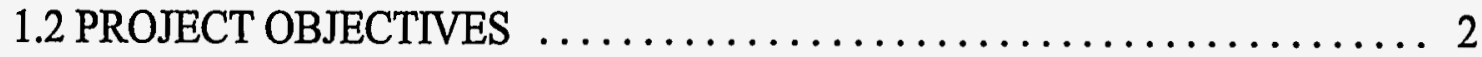

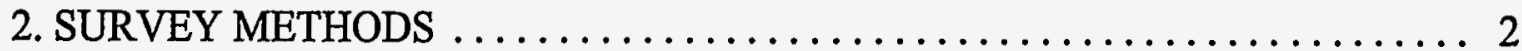

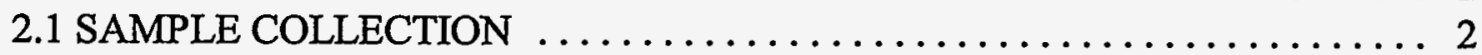

2.2 QUALITY CONTROL $\ldots \ldots \ldots \ldots \ldots \ldots \ldots \ldots \ldots \ldots \ldots \ldots \ldots \ldots$

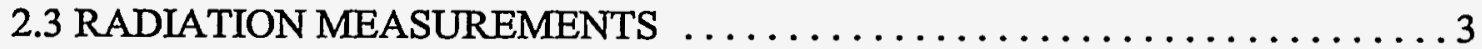

3. SURVEY RESULTS $\ldots \ldots \ldots \ldots \ldots \ldots \ldots \ldots \ldots \ldots \ldots \ldots \ldots \ldots \ldots$

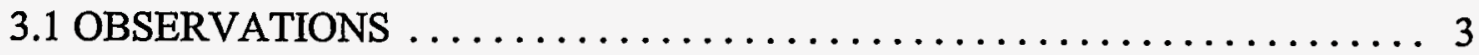

REFERENCES $\ldots \ldots \ldots \ldots \ldots \ldots \ldots \ldots \ldots \ldots \ldots \ldots \ldots \ldots \ldots \ldots \ldots \ldots$

Appendix A. SAMPLING AND ANALYSIS PLAN: SIOU Impoundments

3539 and 3540 Sampling Project $\ldots \ldots \ldots \ldots \ldots \ldots \ldots \ldots \ldots \ldots \ldots \ldots \ldots \ldots \ldots \ldots \ldots \ldots$

Appendix B. HEALTH AND SAFETY ADDENDUM: Sediment Sampling,

Impoundments SIOU 3539 and 3540

B-1 


\section{FIGURES}

1 Location of ponds 3539 and 3540 on the ORNL site $\ldots \ldots \ldots \ldots \ldots \ldots$

2 View of boat operations and pond $3539 \ldots \ldots \ldots \ldots \ldots \ldots \ldots \ldots$

3 Sediment sampling from the boat and view of pond $3540 \ldots \ldots \ldots \ldots \ldots$

4 Sampling locations for the 18-point composites from impoundments 3539

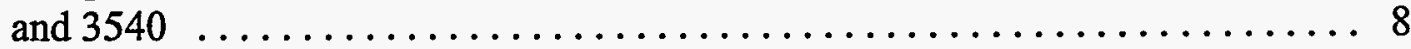

5 Grid sample locations for the east/west composites from impoundments

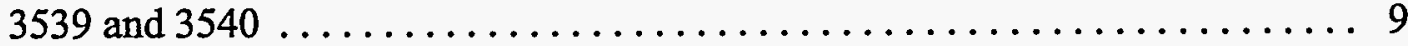




\section{TABLES}

1 Results of analysis of sediment samples taken from impoundment $3539 \ldots \ldots 10$

2 Results of analysis of quality control blank water samples taken during the surface impoundments 3539 and 3540 sampling project $\ldots \ldots \ldots \ldots \ldots 14$

3 Results of analysis of sediment samples taken from impoundment $3540 \ldots \ldots 18$

4 Results of particle-size test on sediment samples from impoundments 3539 and 3540 
-

- 


\section{ACKNOWLEDGMENTS}

This project was sponsored by the U.S. Department of Energy's Office of Environmental Restoration in support of the Oak Ridge National Laboratory (ORNL) Environmental Restoration Program. The authors wish to acknowledge the support of J. D. Kopotic of the Department of Energy, Oak Ridge Operations. The Measurement Applications and Development Group survey team consisted of K. J. Brown, R. L. Coleman, S. P. McKenzie, M. E. Murray, D. E. Rice, R. E. Rodriguez, D. A. Rose, J. Wade, and W. Winton. The authors appreciate the technical and review assistance of V.P. Patania, R. E. Rodriguez, and J. K. Williams of the Life Sciences Division. 


\section{EXECUTIVE SUMMARY}

A radiological and chemical characterization survey of impoundments 3539 and 3540 at the Oak Ridge National Laboratory (ORNL) was conducted during December 1997. Impoundments 3539 and 3540 are located in the Surface Impoundments Operable Unit (SIOU) of Waste Area Group 1. The investigation was performed by the Measurement Applications and Development Group of the Life Sciences Division of ORNL at the request of the Department of Energy (DOE) Office of Environmental Restoration.

Sampling was conducted in order to quantify the presence of polychlorinated biphenyls (PCBs), Resource Conservation and Recovery Act (RCRA) constituents, and other contaminants of interest in support of the Comprehensive Environmental Response, Compensation and Liability Act (CERCLA) remediation for the SIOU.

The survey included collection of sediment/clay samples, quality control blank water samples and equipment rinsate samples for chemical and radiological analysis. Results show the samples contain traces of various organic, inorganic, and radioactive materials. Of particular interest are $P C B$ values which demonstrate the impoundments are not regulated under the Toxic Substances Control Act.

The results for some contaminants of interest are summarized as follows:

\begin{tabular}{lcccc}
\hline \multirow{2}{*}{ Contaminant } & \multicolumn{2}{c}{ Impoundment 3539 } & \multicolumn{2}{c}{ Impoundment 3540 } \\
\cline { 2 - 5 } & Average & Range & Average & Range \\
\hline Total PCBs (ppm) & 5 & $4-8$ & 5 & $3-8$ \\
Total plutonium (pCi/g) & 4 & $1-7$ & 4 & $2-5$ \\
Total uranium (pCi/g) & 11 & $0.4-47$ & 9 & $0.5-22$ \\
Cesium-137 (pCi/g) & 143 & $100-180$ & 68 & $62-78$ \\
Strontium (pCi/g) & 45 & $32-55$ & 51 & $38-67$ \\
Curium-243/244 (pCi/g) & 12 & $8-16$ & 16 & $12-19$ \\
Europium-152 (pCi/g) & 15 & $11-19$ & 11 & $10-13$ \\
TCLP mercury $(\mu \mathrm{g} / \mathrm{L})$ & $<20$ & No range & $<20$ & No range \\
\hline
\end{tabular}




\section{INTRODUCTION}

A radiological and chemical characterization investigation of impoundments 3539 and 3540 at Oak Ridge National Laboratory (ORNL) was conducted during December 1997. The investigation was performed by the Measurement Applications and Development Group of the Life Sciences Division of ORNL at the request of the Department of Energy (DOE) Office of Environmental Restoration. Characterization data obtained from the representative sampling of the sediment/clay materials was used to determine if the impoundments are regulated under the Resource Conservation and Recovery Act (RCRA) or the Toxic Substances Control Act (TSCA) and provide additional information on other potential contaminants.

Impoundments 3539 and 3540 are located in the Surface Impoundments Operable Unit (SIOU) of Waste Area Group (WAG) 1. SIOU consists of four impoundments located in the main plant area of Oak Ridge National Laboratory, just north of White Oak Creek. The two impoundments sampled contain approximately 40 cubic yards of sediment which include organic materials. Figure 1 shows the general location of impoundments 3539 and 3540.

The survey included the following:

- collection of sediment/clay samples for various chemical and radiological analyses,

- collection of quality control blank water samples and equipment rinsate samples for similar analysis, and

- survey of each sediment sample for beta-gamma radiation at the time of collection.

\subsection{SITE HISTORY}

Surface Impoundments Operable Unit (SIOU) was used for management of low-level radioactive wastes generated from experiments and material processing at ORNL. Impoundments 3539 and 3540 were constructed in 1964 and used as a dual-surge basin system designed to alternately receive and discharge process waste streams from the Building 4500 complex.' Process wastes were ultimately discharged into White Oak Creek, following verification of radionuclide content and $\mathrm{pH}$ adjustments. If the waste exceeded the acceptable release limits in place during the period of operations, it was pumped to an adjacent impoundment to be treated at the Process Waste Treatment Plant.

After impoundments 3539 and 3540 were removed from routine service in 1990, they were used occasionally to collect process wastewater when the holding capacity of the current storage tanks for the Process Waste Treatment Plant was exceeded. An additional surge tank was put into service in 1996 to store process wastewater during wet weather. The impoundments were then removed from the waste management emergency service inventory and made available for remediation. 
Impoundments 3539 and 3540 were built by excavation and construction of earthen dikes along the western sides. They are lined with compacted clay and a layer of riprap around the inside slope faces. The impoundments are $100 \mathrm{ft}$ by $58 \mathrm{ft}$, sloping down to $80 \mathrm{ft}$ by $33 \mathrm{ft}$ at the bottom. ${ }^{2}$

\subsection{PROJECT OBJECTIVES}

Sampling was conducted in order to quantify the presence of polychlorinated biphenyls (PCBs), RCRA constituents, and other contaminants of interest in support of the Comprehensive Environmental Response, Compensation and Liability Act (CERCLA) remediation for the SIOU.

\section{SURVEY METHODS}

\subsection{SAMPLE COLLECTION}

A description of sampling methods is provided in "Sampling and Analysis Plan: SIOU Impoundments 3539 and 3540 Sampling Project" (SAP, see Appendix A). Health and safety support was provided as specified in "Health and Safety Addendum: Sediment Sampling, Impoundments SIOU 3539 and 3540" (see Appendix B), a project-specific addendum to the original health and safety plan, "WAG 1 Surface Impoundments Operable Unit (SIOU) Treatability Study," SSHASP No 001-226/0010 0396.

Sediment/clay samples were collected from the bottom of each pond using a decontaminated coring sampler, as described in LMES procedure Collection of Sediment Samples, ESP-304-1. The stainless steel push corer was inserted into the sediment and removed manually. A fiat-bottomed boat and walkboards were used to gain access to some sampling locations. Three composite samples were obtained from 36 locations in each pond. One composite sample consisted of sample media from 18 locations, using a statistically generated 2-feet by 2-feet grid of random numbered locations. Alternate points on the grid were sampled if some of the first 18 locations were unsuitable to obtain a representative core of the pond bottom (i.e., top of riprap, in concrete sump). The second and third composite samples were collected from systematic locations on the grid, which resulted in the collection of nine samples from the west side of the pond and nine from the east side of the pond. The sampled media contained organic materials such as leaves, small twigs, decomposed organic materials, and about 0.1 foot of clay.

Each composite sample was homogenized, then bottled, labeled and preserved according to specifications of the SAP and Quality Assurance Plan for the Oak Ridge National Laboratory Environmental Restoration Program, ORNL/ER-225/R1. Samples, accompanied by chain-of-custody forms, were taken to the ORNL Analytical Services Organization for chemical and radionuclide analysis. 


\subsection{QUALITY CONTROL}

Field quality control samples were taken during the project. The composite sample taken from 18 random locations in Impoundment 3539 was split for separate analyses. Two field blank water samples were taken, at the beginning and end of sampling activities, respectively. An equipment rinsate blank was taken from each impoundment. Quality control samples were analyzed according to the SAP.

A quality assurance field surveillance was conducted on December 11, 1997 to verify that field activities were being conducted in accordance with the sampling and analysis plan. One positive and three negative observations were noted in Environmental Management and Enrichment Facilities Field Surveillance Report \#97SIOU-1 dated December 16, 1997. The observations noted, however, were not related to and had no impact on the quality of samples or the data obtained for this project. The observations addressed administrative and documentation deficiencies.

\subsection{RADIATION MEASUREMENTS}

A description of typical methods and instrumentation providing guidance for the conduct of the radiological aspects of the survey is presented in Procedures Manual for the ORNL Radiological Survey Activities (RASA) Program, ORNL/TM-8600 (April 1987) ${ }^{3}$ and Measurement Applications and Development Group Guidelines, ORNL-6782 (January 1995). ${ }^{4}$

Sediment samples were scanned for beta-gamma radiation at the time of collection using a portable Bicron Analyst scaler/ratemeter with an Eberline HP-210 Geiger-Mueller pancake detector $\left(<2 \mathrm{mg} / \mathrm{cm}^{2}\right.$ wall thickness) with fine mesh screen. The purpose of this check was to observe any anomalies in contamination distribution and for contamination control.

Prior to exiting the site, survey personnel were checked for beta-gamma contamination. A Bicron Analyst meter with an HP-260 probe ("pancake") was used to check for betagamma contamination.

\subsection{OBSERVATIONS}

\section{SURVEY RESULTS}

The sampling and analysis effort determined the average concentrations for the TSCA, RCRA and other contaminants of interest. Sampling locations at impoundments 3539 and 3540 for the 18-point composite are shown in Fig. 4. The east/west composite sampling locations are shown in Fig. 5.

Tables 1-4 show the results of chemical and radionuclide analysis of samples and quality control samples taken from Impoundments 3539 and 3540 . Results show the samples contain traces of various organic, inorganic, and radioactive materials. Of particular interest 
are the PCB values (less than $50 \mathrm{ppm}$ ), which demonstrate the impoundments not to be TSCA-regulated.

\section{REFERENCES}

1. Site Characterization Summary Report for Waste Area Grouping 1 at Oak Ridge National Laboratory, Oak Ridge, Tennessee, DOE/OR-1043/V1-V5\&D1. U.S. Department of Energy, Oak Ridge, TN.

2. Remedial Investigation/Feasibility Study for Surface Impoundments Operable Unit Waste Area Grouping 1, Oak Ridge National Laboratory, Oak Ridge, Tennessee, DOE/OR/02-1346\&D2, Jacobs ER Team and Lockheed Martin Energy Systems, Inc., Oak Ridge, Tennessee, November 1995.

3. T. E. Myrick, B. A. Berven, W. D. Cottrell, W. A. Goldsmith, and F. F. Haywood, Procedures Manual for the ORNL Radiological Survey Activities (RASA) Program, ORNL/TM-8600, Martin Marietta Energy Systems, Inc., Oak Ridge Natl. Lab., April 1987.

4. Oak Ridge National Laboratory, Health Sciences Research Division, Measurement Applications and Development Group Guidelines, ORNL-6782, Martin Marietta Energy Systems, Inc., January 1995. 
ORNL-DWG 98-4156

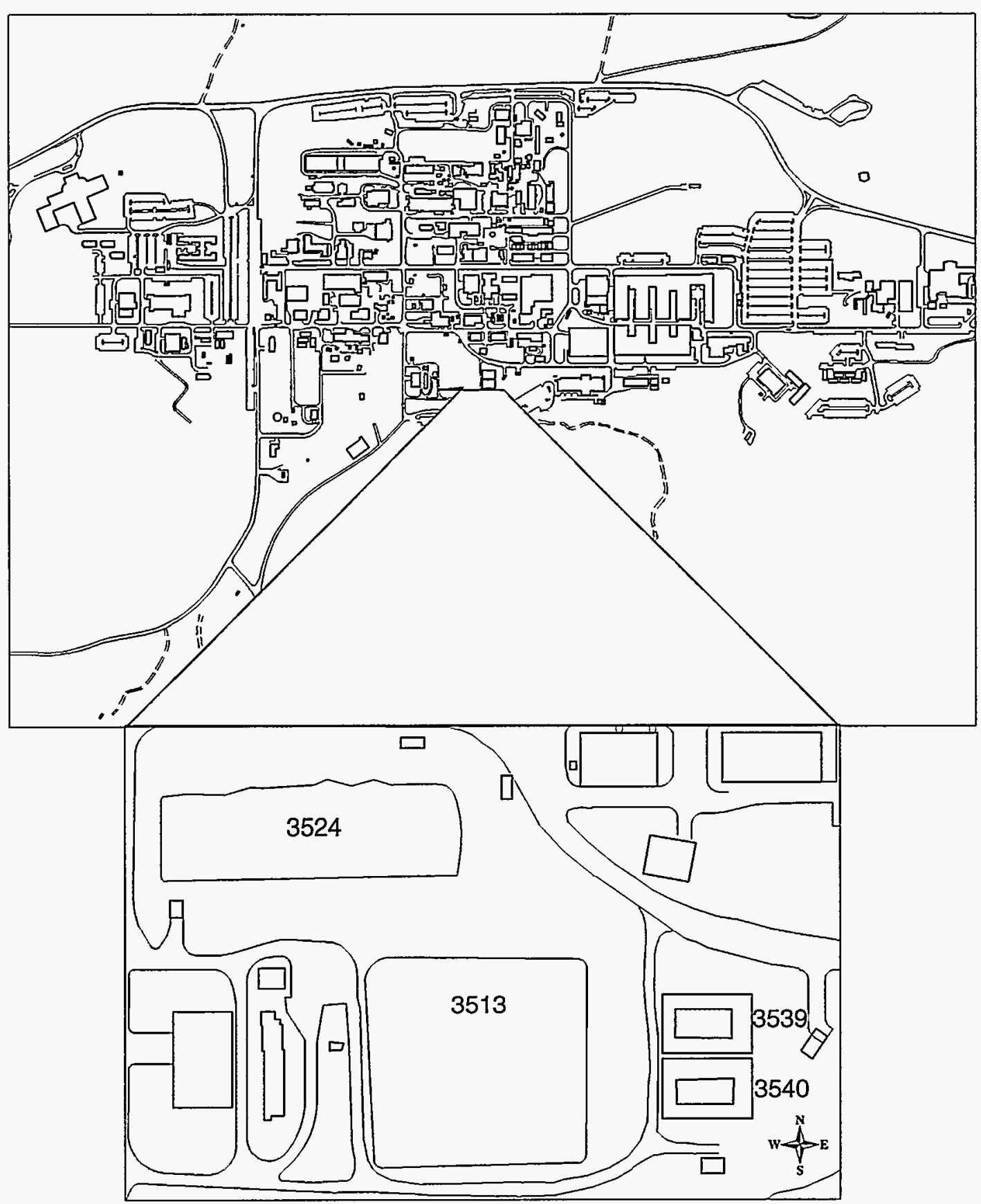

Fig. 1. Location of ponds 3539 and 3540 on the ORNL site. 
ORNL-PHOTO 1161-98

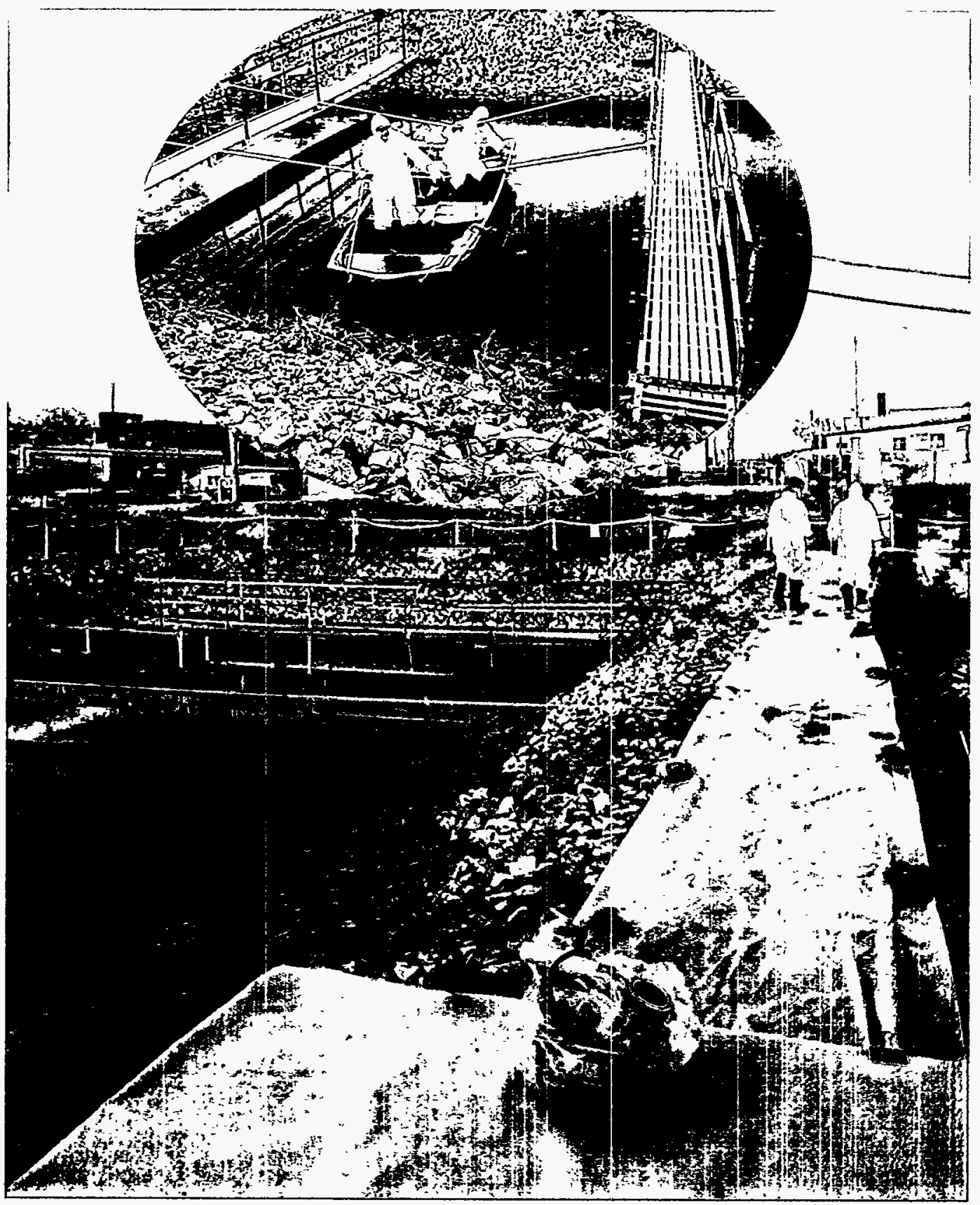

Fig. 2. View of boat operations and pond 3539. 
ORNL-PHOTO 1162-98

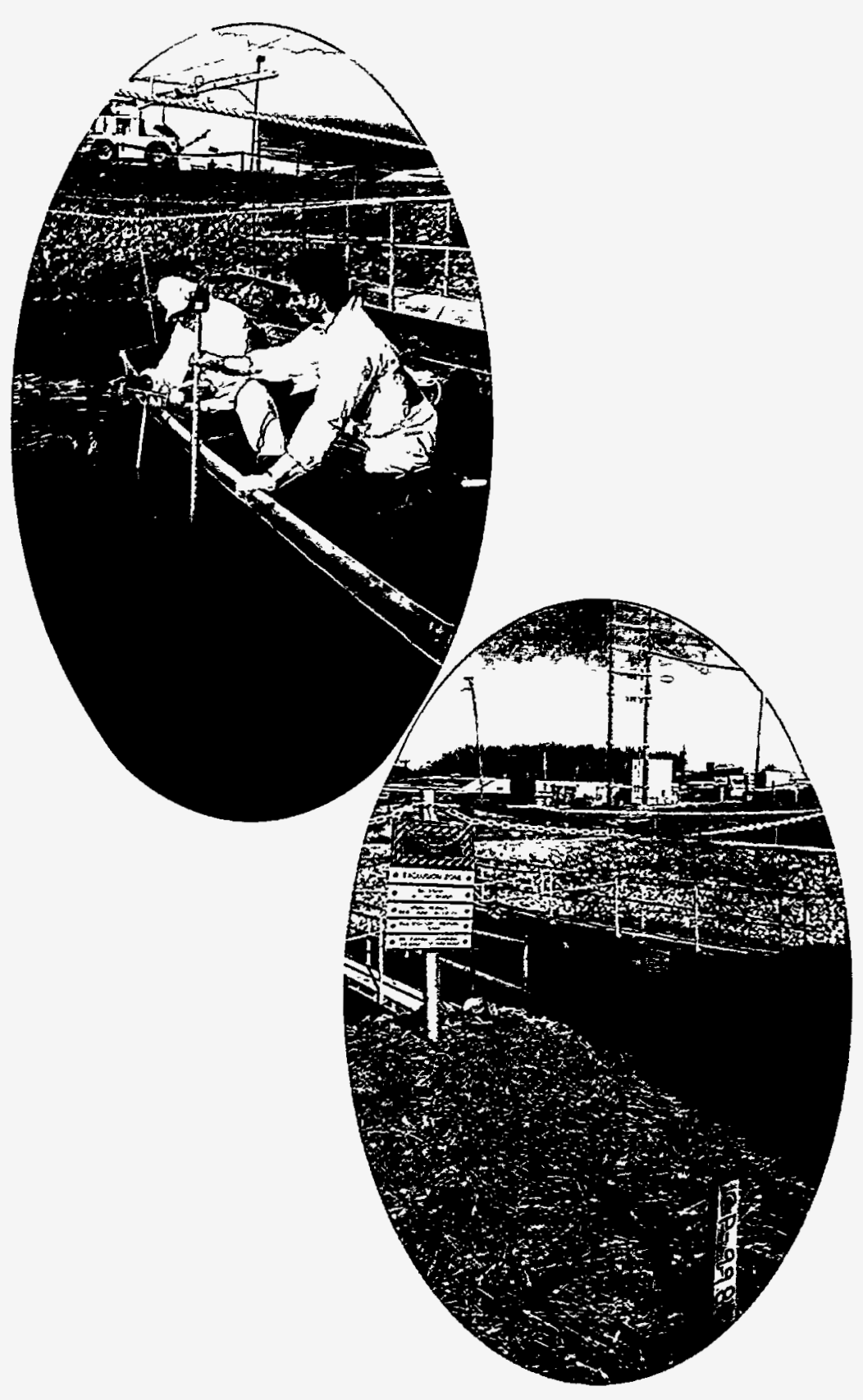

Fig. 3. Sediment sampling from the boat and view of pond 3540 . 
ORNL-DWG 98-4157

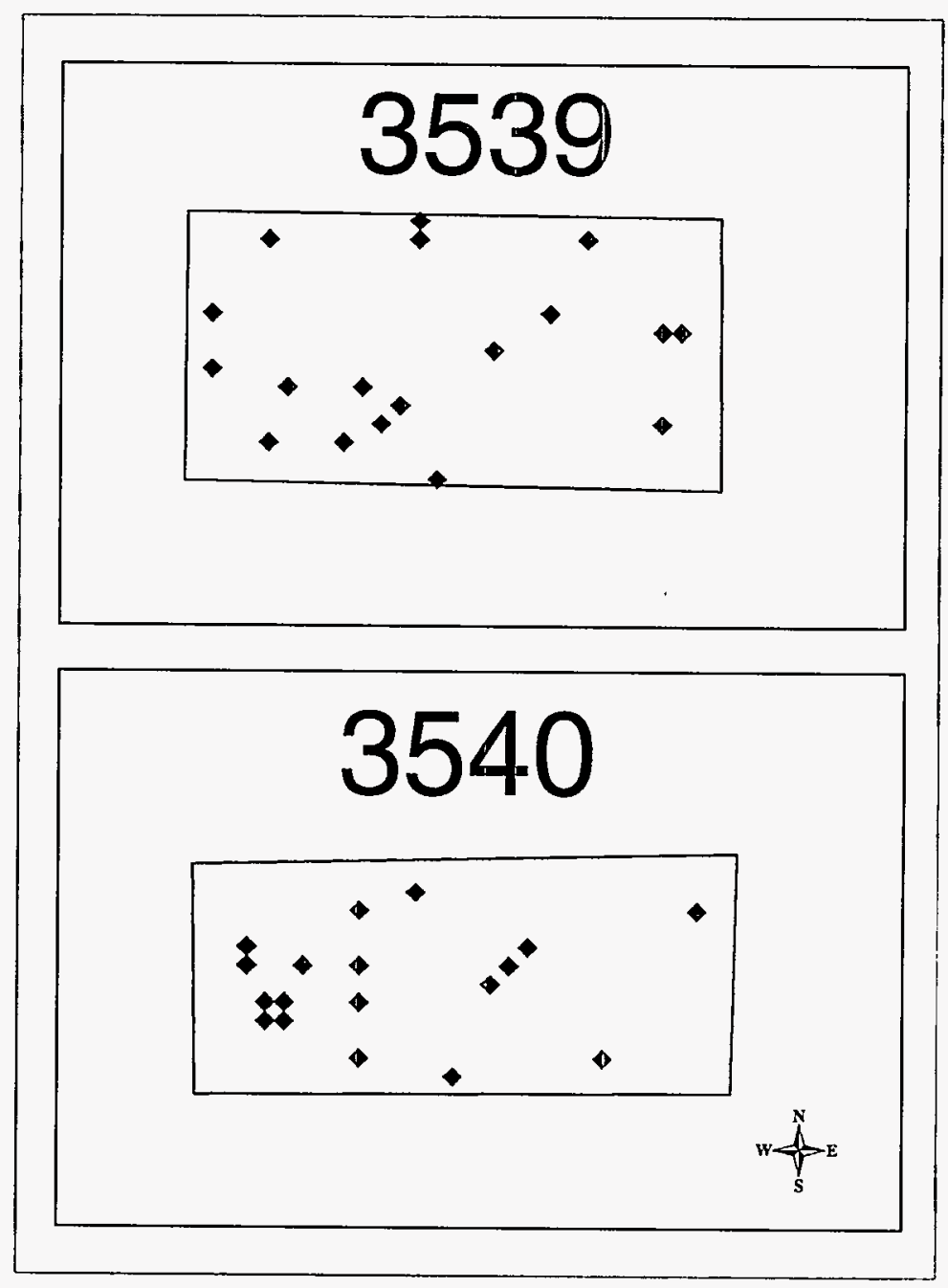

Fig. 4. Sampling locations for the 18-point composites from impoundments 3539 and 3540 . Locations may vary from SAP due to accessibility (see Sect. 6.3.2 of SAP). 
ORNL-DWG 98-4158

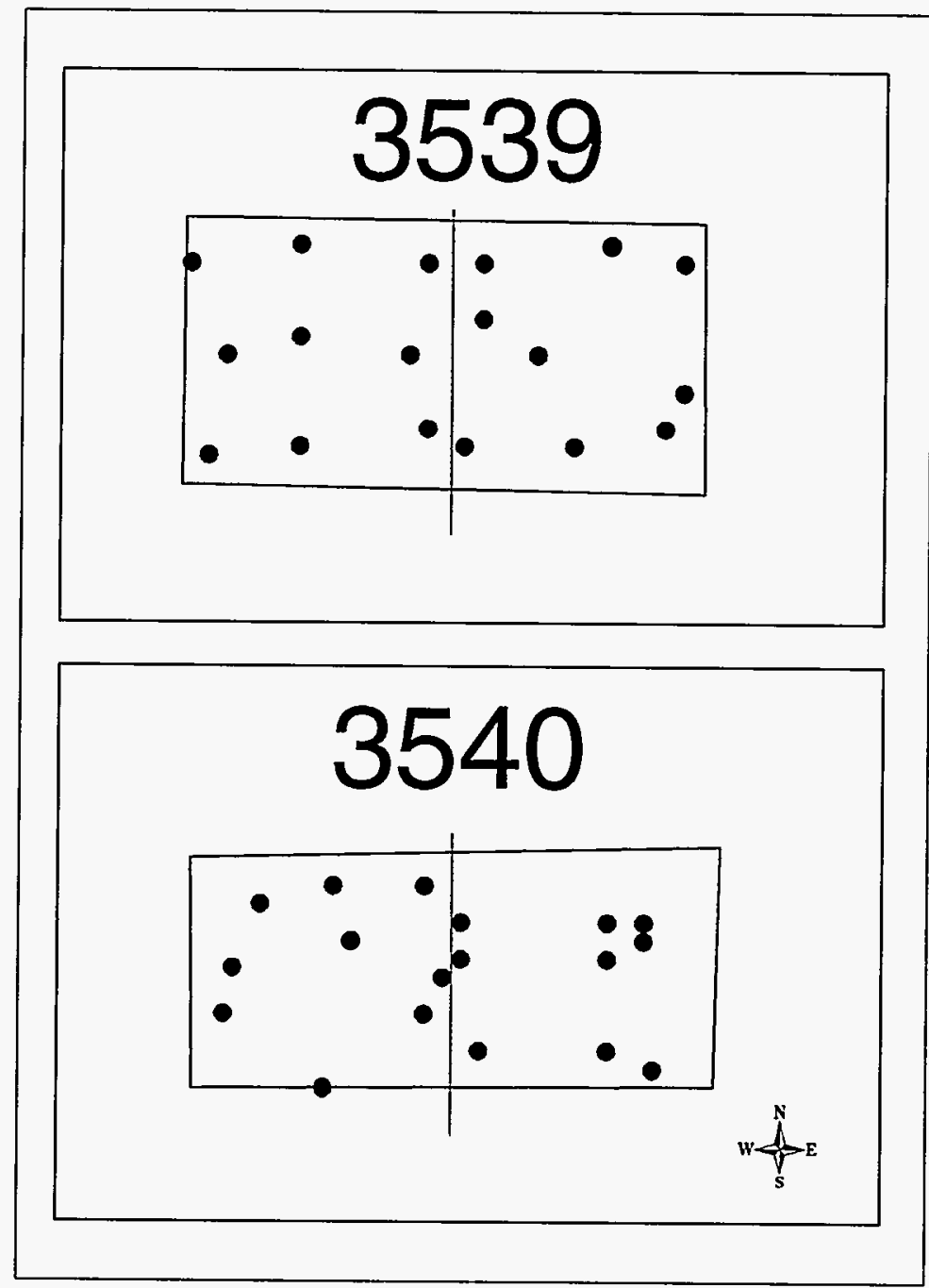

Fig. 5. Grid sample locations for the east/west composites from impoundments 3539 and 3540. 
Table 1. Results of analysis of sediment samples taken from impoundment 3539

\begin{tabular}{|c|c|c|c|c|c|}
\hline Analyte & Unit $^{a}$ & SIOU39COMP1 ${ }^{b}$ & SIOU39WEST ${ }^{c}$ & SIOU39EAST ${ }^{d}$ & 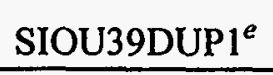 \\
\hline \multicolumn{6}{|c|}{ TCLP for: Semi-Volatiles, Metals, Pesticides, Herbicides } \\
\hline Antimony & $\mathrm{mg} / \mathrm{L}$ & $<0.40$ & $<0.40$ & $<0.40$ & $<0.40$ \\
\hline Arsenic & $\mathrm{mg} / \mathrm{L}$ & $<0.40$ & $<0.40$ & $<0.40$ & $<0.40$ \\
\hline Barium & $\mathrm{mg} / \mathrm{L}$ & $<1.0$ & 1.2 & $<1.0$ & 1.0 \\
\hline Beryllium & $\mathrm{mg} / \mathrm{L}$ & $<0.0020$ & 0.0020 & $<0.0020$ & $<0.0020$ \\
\hline Cadmium & $\mathrm{mg} / \mathrm{L}$ & 0.071 & 0.10 & 0.24 & 0.065 \\
\hline Chromium & $\mathrm{mg} / \mathrm{L}$ & $<0.040$ & $<0.040$ & $<0.040$ & $<0.040$ \\
\hline Copper & $\mathrm{mg} / \mathrm{L}$ & 0.81 & 1.56 & $<0.040$ & 0.74 \\
\hline Lead & $\mathrm{mg} / \mathrm{L}$ & 2.8 & 3.4 & $<0.20$ & 2.5 \\
\hline Nickel & $\mathrm{mg} / \mathrm{L}$ & 0.27 & 0.41 & $<0.10$ & 0.30 \\
\hline Silver & $\mathrm{mg} / \mathrm{L}$ & $<0.040$ & $<0.040$ & $<0.040$ & $<0.040$ \\
\hline Vanadium & $\mathrm{mg} / \mathrm{L}$ & $<0.040$ & $<0.040$ & $<0.040$ & $<0.040$ \\
\hline Zinc & $\mathrm{mg} / \mathrm{L}$ & 3.4 & 3.3 & 7.7 & 3.1 \\
\hline Mercury & $\mu \mathrm{g} / \mathrm{L}$ & $<20$ & $<20$ & $<20$ & $<20$ \\
\hline Selenium & $\mu \mathrm{g} / \mathrm{L}$ & $<20$ & $<20$ & $<20$ & $<20$ \\
\hline $2,4-D$ & $\mu \mathrm{g} / \mathrm{L}$ & $<4$ & $<4$ & $<4$ & $<4$ \\
\hline Silvex & $\mu \mathrm{g} / \mathrm{L}$ & $<4$ & $<4$ & $<4$ & $<4$ \\
\hline Chlordane & $\mu \mathrm{g} / \mathrm{L}$ & $<13$ & $<13$ & $<13$ & $<13$ \\
\hline Endrin & $\mu \mathrm{g} / \mathrm{L}$ & $<1.6$ & $<1.6$ & $<1.6$ & $<1.6$ \\
\hline Heptachlor & $\mu \mathrm{g} / \mathrm{L}$ & $<0.8$ & $<0.8$ & $<0.8$ & $<0.8$ \\
\hline Heptachlor epoxide & $\mu \mathrm{g} / \mathrm{L}$ & $<0.8$ & $<0.8$ & $<0.8$ & $<0.8$ \\
\hline Lindane & $\mu \mathrm{g} / \mathrm{L}$ & $<0.8$ & $<0.8$ & $<0.8$ & $<0.8$ \\
\hline
\end{tabular}


Table 1 (continued)

\begin{tabular}{|c|c|c|c|c|c|}
\hline Analyte & $\mathrm{Unit}^{a}$ & SIOU $39 C O M P 1^{b}$ & SIOU39WEST ${ }^{c}$ & SIOU39EAST $^{d}$ & 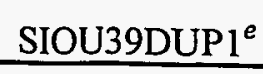 \\
\hline Methoxychlor & $\mu \mathrm{g} / \mathrm{L}$ & $<8$ & $<8$ & $<8$ & $<8$ \\
\hline Toxaphene & $\mu \mathrm{g} / \mathrm{L}$ & $<13$ & $<13$ & $<13$ & $<13$ \\
\hline 1,4-Dichlorobenzene & $\mu \mathrm{g} / \mathrm{kg}$ & $<10$ & $<10$ & $<10$ & $<10$ \\
\hline 2,4,5-Trichlorophenol & $\mu \mathrm{g} / \mathrm{kg}$ & $<10$ & $<10$ & $<10$ & $<10$ \\
\hline 2,4,6-Trichlorophenol & $\mu \mathrm{g} / \mathrm{kg}$ & $<10$ & $<10$ & $<10$ & $<10$ \\
\hline 2,4-Dinitrotoluene & $\mu \mathrm{g} / \mathrm{kg}$ & $<10$ & $<10$ & $<10$ & $<10$ \\
\hline 2-Methylphenol & $\mu \mathrm{g} / \mathrm{kg}$ & $<10$ & $<10$ & $<10$ & $<10$ \\
\hline 3- and 4- Methylphenol & $\mu \mathrm{g} / \mathrm{kg}$ & $<10$ & $<10$ & $<10$ & $<10$ \\
\hline Hexachlorobenzene & $\mu \mathrm{g} / \mathrm{kg}$ & $<10$ & $<10$ & $<10$ & $<10$ \\
\hline Hexachlorobutadiene & $\mu \mathrm{g} / \mathrm{kg}$ & $<10$ & $<10$ & $<10$ & $<10$ \\
\hline Hexachloroethane & $\mu \mathrm{g} / \mathrm{kg}$ & $<10$ & $<10$ & $<10$ & $<10$ \\
\hline Nitrobenzene & $\mu \mathrm{g} / \mathrm{kg}$ & $<10$ & $<10$ & $<10$ & $<10$ \\
\hline Pentachlorophenol & $\mu \mathrm{g} / \mathrm{kg}$ & $<10$ & $<10$ & $<10$ & $<10$ \\
\hline Pyridine & $\mu \mathrm{g} / \mathrm{kg}$ & $<10$ & $<10$ & $<10$ & $<10$ \\
\hline \multicolumn{6}{|c|}{ PCBs (Total and Aroclor-Specific) } \\
\hline Aroclor-1016 & $\mu \mathrm{g} / \mathrm{kg}$ & $<490$ & $<2200$ & $<360$ & $<480$ \\
\hline Aroclor-1221 & $\mu \mathrm{g} / \mathrm{kg}$ & $<490$ & $<2200$ & $<360$ & $<480$ \\
\hline Aroclor- 1232 & $\mu \mathrm{g} / \mathrm{kg}$ & $<490$ & $<2200$ & $<360$ & $<480$ \\
\hline Aroclor-1242 & $\mu \mathrm{g} / \mathrm{kg}$ & $<490$ & $<2200$ & $<360$ & $<480$ \\
\hline Aroclor -1248 & $\mu \mathrm{g} / \mathrm{kg}$ & $<490$ & $<2200$ & $<360$ & $<480$ \\
\hline Aroclor-1254 & $\mu \mathrm{g} / \mathrm{kg}$ & 7600 & 3600 & 4000 & 5900 \\
\hline Aroclor -1260 & $\mu \mathrm{g} / \mathrm{kg}$ & $<490$ & $<2200$ & $<360$ & $<480$ \\
\hline
\end{tabular}


Table 1 (continued)

\begin{tabular}{|c|c|c|c|c|c|}
\hline Analyte & Unit $^{a}$ & SIOU39COMPI ${ }^{b}$ & SIOU39WEST $^{c}$ & SIOU39EAST ${ }^{d}$ & SIOU39DUP $1^{e}$ \\
\hline PCB, Total & $\mu \mathrm{g} / \mathrm{kg}$ & 7600 & 3600 & 4000 & 5900 \\
\hline \multicolumn{6}{|c|}{ Reactive Cyanide, Reactive Sulfide, Oil and Grease, Total Organic Halogen (TOX) } \\
\hline Cyanide & $\mathrm{mg} / \mathrm{kg}$ & $<50$ & $<50$ & $<50$ & $<50$ \\
\hline Hydrogen Sulfide & $\mathrm{mg} / \mathrm{kg}$ & 125 & 70 & 110 & 200 \\
\hline Oil and Grease & $\mu \mathrm{g} / \mathrm{g}$ & 700 & 490 & 650 & 1300 \\
\hline $\begin{array}{l}\text { Total Organic Halides } \\
\text { (TOX) }\end{array}$ & $\mu \mathrm{g} / \mathrm{g}$ & $<25$ & $<25$ & $<25$ & $<25$ \\
\hline \multicolumn{6}{|c|}{ Radionuclides (dry weight basis) } \\
\hline Americium-241 & $\mathrm{pCi} / \mathrm{g}$ & $2.1 \pm 0.25$ & $1.7 \pm 0.21$ & $0.96 \pm 0.16$ & $1.9 \pm 0.22$ \\
\hline Curium-243/244 & $\mathrm{pCi} / \mathrm{g}$ & $16 \pm 1.2$ & $8.0 \pm 0.65$ & $8.2 \pm 0.70$ & $14 \pm 1.1$ \\
\hline Neptunium-237 & $\mathrm{pCi} / \mathrm{g}$ & $0.015 \pm 0.042$ & $0.011 \pm 0.037$ & $0.018 \pm 0.035$ & $0.039 \pm 0.059$ \\
\hline Plutonium-238 & $\mathrm{pCi} / \mathrm{g}$ & $6.9 \pm 1.3$ & $4.2 \pm 0.8$ & $3.2 \pm 0.6$ & $5.5 \pm 1.1$ \\
\hline Plutonium-239/240 & $\mathrm{pCi} / \mathrm{g}$ & $2.7 \pm 0.6$ & $2.0 \pm 0.4$ & $1.3 \pm 0.3$ & $2.5 \pm 0.6$ \\
\hline Thorium-228 & $\mathrm{pCi} / \mathrm{g}$ & $16 \pm 4$ & $16 \pm 4$ & $9.1 \pm 2.3$ & $16 \pm 4$ \\
\hline Thorium-230 & $\mathrm{pCi} / \mathrm{g}$ & $2.2 \pm 0.1$ & $1.2 \pm 0.42$ & $1.2 \pm 0.43$ & $1.8 \pm 0.6$ \\
\hline Thorium-232 & $\mathrm{pCi} / \mathrm{g}$ & $13 \pm 3$ & $13 \pm 4$ & $9.0 \pm 2.5$ & $14 \pm 4$ \\
\hline Uranium-233 ${ }^{f}$ & $\mathrm{pCi} / \mathrm{g}$ & 6 & 3 & 3 & 6 \\
\hline Uranium-234 & $\mathrm{pCi} / \mathrm{g}$ & $24 \pm 4$ & $16 \pm 3$ & $14 \pm 3$ & $47 \pm 9$ \\
\hline Uranium-235 & $\mathrm{pCi} / \mathrm{g}$ & $0.67 \pm 0.23$ & $0.52 \pm 0.19$ & $0.44 \pm 0.19$ & $1.0 \pm 0.3$ \\
\hline Uranium-238 & $\mathrm{pCi} / \mathrm{g}$ & $9.4 \pm 1.8$ & $7.0 \pm 1.4$ & $5.4 \pm 1.2$ & $9.0 \pm 1.9$ \\
\hline Carbon-14 & $\mathrm{pCi} / \mathrm{g}$ & $38.8 \pm 25$ & $36.4 \pm 24$ & $27.3 \pm 22$ & $94.0 \pm 23$ \\
\hline Cesium-137 & $\mathrm{pCi} / \mathrm{g}$ & $180 \pm 17$ & $130 \pm 13$ & $100 \pm 6.3$ & $160 \pm 19$ \\
\hline Cobalt- 60 & $\mathrm{pCi} / \mathrm{g}$ & $1.8 \pm 0.17$ & $1.5 \pm 0.18$ & $0.93 \pm 0.12$ & $1.5 \pm 0.16$ \\
\hline
\end{tabular}


Table 1 (continued)

\begin{tabular}{lccccc}
\hline Analyte & Unit $^{a}$ & SIOU39COMP $^{b}$ & SIOU39WEST $^{c}$ & SIOU39EAST $^{d}$ & ${\text { SIOU39DUP }{ }^{e}}^{c}$ \\
\hline Europium-152 & $\mathrm{pCi} / \mathrm{g}$ & $19 \pm 1.3$ & $13 \pm 0.87$ & $11 \pm 0.75$ & $16 \pm 1.0$ \\
Europium-154 & $\mathrm{pCi} / \mathrm{g}$ & $4.5 \pm 0.58$ & $4.0 \pm 0.60$ & $3.1 \pm 0.58$ & $3.9 \pm 0.35$ \\
Europium-155 & $\mathrm{pCi} / \mathrm{g}$ & $1.6 \pm 0.66$ & $2.2 \pm 0.58$ & $3.2 \pm 0.64$ & $4.3 \pm 0.87$ \\
Osmium-191 & $\mathrm{pCi} / \mathrm{g}$ & $3.2 \pm 1.3$ & $1.4 \pm 1.0$ & $1.7 \pm 0.85$ & $2.6 \pm 1.1$ \\
Potassium-40 & $\mathrm{pCi} / \mathrm{g}$ & $11 \pm 2.3$ & $11 \pm 2.2$ & $8.2 \pm 2.0$ & $12 \pm 2.1$ \\
Alpha activity & $\mathrm{pCi} / \mathrm{g}$ & $97 \pm 13$ & $100 \pm 10$ & $76 \pm 11$ & $13 \pm 20$ \\
Beta activity & $\mathrm{pCi} / \mathrm{g}$ & $340 \pm 19$ & $280 \pm 20$ & $170 \pm 10$ & $360 \pm 20$ \\
Technetium-99 & $\mathrm{pCi} / \mathrm{g}$ & $14 \pm 2$ & $14 \pm 2$ & $6.7 \pm 0.4$ & $32 \pm 1$ \\
Strontium-90 & $\mathrm{pCi} / \mathrm{g}$ & $45 \pm 2$ & $46 \pm 2$ & $165 \pm 95$ & $55 \pm 2$ \\
Tritium & $\mathrm{pCi} / \mathrm{g}$ & $<168$ & $475 \pm 110$ & $<143$ \\
\hline
\end{tabular}

${ }^{a}$ Conversion factors: $\mathrm{mg} / \mathrm{L}=\mathrm{ppm}, \mu \mathrm{g} / \mathrm{L}=\mathrm{ppb}, \mu \mathrm{g} / \mathrm{g}=\mathrm{ppm}, \mathrm{mg} / \mathrm{kg}=\mathrm{ppm}, \mu \mathrm{g} / \mathrm{kg}=\mathrm{ppb}$.

${ }^{b}$ SIOU39COMP1 corresponds to Lab Sample ID A97345006 and Chain of Custody Number 103958.

${ }^{c}$ SIOU39WEST corresponds to Lab Sample ID A973490044 and Chain of Custody Number 103960.

${ }^{d}$ SIOU39EAST corresponds to Lab Sample ID A973460109 and Chain of Custody Number 103959.

${ }^{e}$ SIOU39DUP1 corresponds to Lab Sample ID A973450007and Chain of Custody Number 103958.

${ }^{f}$ Uranium-233 values are estimated based on visual observations of the spectra by the authors. ASO documentation qualifies $U-233$ present but does not quantify any values. 
Table 2. Results of analysis of quality control blank water samples taken during the impoundments 3539 and 3540 sampling project

\begin{tabular}{|c|c|c|c|c|c|}
\hline Analyte & Unit $^{a}$ & SIOUFB $1^{b}$ & SIOUFB2 $^{c}$ & SIOU39ERB $1^{d}$ & SIOU40ERB $1^{e}$ \\
\hline \multicolumn{6}{|c|}{ TCLP for: Semi-Volatiles, Metals, Pesticides, Herbicides } \\
\hline Antimony & $\mathrm{mg} / \mathrm{L}$ & $<0.40$ & $<0.40$ & $<0.40$ & $<0.40$ \\
\hline Arsenic & $\mathrm{mg} / \mathrm{L}$ & $<0.40$ & $<0.40$ & $<0.40$ & $<0.40$ \\
\hline Barium & $\mathrm{mg} / \mathrm{L}$ & $<1.0$ & $<1.0$ & $<1.0$ & $<1.0$ \\
\hline Beryllium & $\mathrm{mg} / \mathrm{L}$ & $<0.0020$ & $<0.0020$ & $<0.0020$ & $<0.0020$ \\
\hline Cadmium & $\mathrm{mg} / \mathrm{L}$ & $<0.020$ & $<0.020$ & $<0.020$ & $<0.020$ \\
\hline Chromium & $\mathrm{mg} / \mathrm{L}$ & $<0.040$ & $<0.040$ & $<0.040$ & $<0.040$ \\
\hline Copper & $\mathrm{mg} / \mathrm{L}$ & $<0.040$ & $<0.040$ & $<0.040$ & $<0.040$ \\
\hline Lead (by ICPAES) & $\mathrm{mg} / \mathrm{L}$ & $<0.20$ & $<0.20$ & $<0.20$ & $<0.20$ \\
\hline Nickel & $\mathrm{mg} / \mathrm{L}$ & $<0.10$ & $<0.10$ & $<0.10$ & $<0.10$ \\
\hline Silver & $\mathrm{mg} / \mathrm{L}$ & $<0.040$ & $<0.040$ & $<0.040$ & $<0.040$ \\
\hline Vanadium & $\mathrm{mg} / \mathrm{L}$ & $<0.040$ & $<0.040$ & $<0.040$ & $<0.040$ \\
\hline Zinc & $\mathrm{mg} / \mathrm{L}$ & $<1.0$ & $<1.0$ & $<1.0$ & $<1.0$ \\
\hline Mercury & $\mu \mathrm{g} / \mathrm{L}$ & $<20$ & $<20$ & $<20$ & $<20$ \\
\hline Lead (by GFAA) & $\mu \mathrm{g} / \mathrm{L}$ & $<10$ & $<10$ & $<10$ & $<12.6$ \\
\hline Selenium & $\mu \mathrm{g} / \mathrm{L}$ & $<20$ & $<20$ & $<20$ & $<20$ \\
\hline $2,4-\mathrm{D}$ & $\mu \mathrm{g} / \mathrm{L}$ & $<120$ & $<4$ & $<4$ & $<4$ \\
\hline Silvex & $\mu \mathrm{g} / \mathrm{L}$ & $<40$ & $<4$ & $<4$ & $<4$ \\
\hline Chlordane & $\mu \mathrm{g} / \mathrm{L}$ & $<10$ & $<13$ & $<13$ & $<13$ \\
\hline Endrin & $\mu \mathrm{g} / \mathrm{L}$ & $<2$ & $<1.6$ & $<1.6$ & $<1.6$ \\
\hline Heptachlor & $\mu \mathrm{g} / \mathrm{L}$ & $<1$ & $<0.8$ & $<0.8$ & $<0.8$ \\
\hline Heptachlor epoxide & $\mu \mathrm{g} / \mathrm{L}$ & $<1$ & $<0.8$ & $<0.8$ & $<0.8$ \\
\hline
\end{tabular}


Table 2 (continued)

\begin{tabular}{|c|c|c|c|c|c|}
\hline Analyte & Unit $^{a}$ & SIOUFB1 $^{b}$ & SIOUFB2 $^{c}$ & SIOU39ERB $1^{d}$ & SIOU40ERB1 $1^{e}$ \\
\hline Lindane & $\mu \mathrm{g} / \mathrm{L}$ & $<1$ & $<0.8$ & $<0.8$ & $<0.8$ \\
\hline Methoxychlor & $\mu \mathrm{g} / \mathrm{L}$ & $<10$ & $<8$ & $<8$ & $<8$ \\
\hline Toxaphene & $\mu \mathrm{g} / \mathrm{L}$ & $<50$ & $<13$ & $<13$ & $<13$ \\
\hline 1,4-Dichlorobenzene & $\mu \mathrm{g} / \mathrm{L}$ & $<40$ & $<10$ & $<11$ & $<10$ \\
\hline 2,4,5-Trichlorophenol & $\mu \mathrm{g} / \mathrm{L}$ & $<40$ & $<10$ & $<11$ & $<10$ \\
\hline 2,4,6-Trichlorophenol & $\mu \mathrm{g} / \mathrm{L}$ & $<40$ & $<10$ & $<11$ & $<10$ \\
\hline 2,4-Dinitrotoluene & $\mu \mathrm{g} / \mathrm{L}$ & $\cdot<40$ & $<10$ & $<11$ & $<10$ \\
\hline 2-Methylphenol & $\mu \mathrm{g} / \mathrm{L}$ & $<40$ & $<10$ & $<11$ & $<10$ \\
\hline 3- and 4-Methylphenol & $\mu \mathrm{g} / \mathrm{L}$ & $<40$ & $<10$ & $<11$ & $<10$ \\
\hline Hexachlorobenzene & $\mu \mathrm{g} / \mathrm{L}$ & $<40$ & $<10$ & $<11$ & $<10$ \\
\hline Hexachlorobutadiene & $\mu \mathrm{g} / \mathrm{L}$ & $<40$ & $<10$ & $<11$ & $<10$ \\
\hline Hexachloroethane & $\mu \mathrm{g} / \mathrm{L}$ & $<40$ & $<10$ & $<11$ & $<10$ \\
\hline Nitrobenzene & $\mu \mathrm{g} / \mathrm{L}$ & $<40$ & $<10$ & $<11$ & $<10$ \\
\hline Pentachlorophenol & $\mu \mathrm{g} / \mathrm{L}$ & $<40$ & $<10$ & $<11$ & $<10$ \\
\hline Pyridine & $\mu \mathrm{g} / \mathrm{L}$ & $<80$ & $<20$ & $<11$ & $<20$ \\
\hline \multicolumn{6}{|c|}{ PCBs (Total and Aroclor-Specific) } \\
\hline Aroclor-1016 & $\mu \mathrm{g} / \mathrm{L}$ & 0.5 & 50 & 50 & 50 \\
\hline Aroclor-1221 & $\mu \mathrm{g} / \mathrm{L}$ & 0.5 & 50 & 50 & 50 \\
\hline Aroclor-1232 & $\mu \mathrm{g} / \mathrm{L}$ & 0.5 & 50 & 50 & 50 \\
\hline Aroclor-1242 & $\mu \mathrm{g} / \mathrm{L}$ & 0.5 & 50 & 50 & 50 \\
\hline Aroclor-1248 & $\mu \mathrm{g} / \mathrm{L}$ & 0.5 & 50 & 50 & 50 \\
\hline Aroclor-1254 & $\mu \mathrm{g} / \mathrm{L}$ & 0.2 & 50 & 50 & 50 \\
\hline
\end{tabular}


Table 2 (continued)

\begin{tabular}{|c|c|c|c|c|c|}
\hline Analyte & Unit $^{a}$ & SIOUFB $^{b}$ & SIOUFB2 ${ }^{c}$ & SIOU39ERB $1^{d}$ & SIOU $40 \mathrm{ERB} 1^{e}$ \\
\hline Aroclor- 1260 & $\mu \mathrm{g} / \mathrm{L}$ & 0.5 & 50 & 50 & 50 \\
\hline PCB, Total & $\mu \mathrm{g} / \mathrm{L}$ & 0.2 & 50 & 50 & 50 \\
\hline \multicolumn{6}{|c|}{ Reactive Cyanide, Reactive Sulfide, Oil and Grease, Total Organic Carbon (TOC), Total Organic Halogen (TOX) } \\
\hline Cyanide & $\mathrm{mg} / \mathrm{kg}$ & $<50$ & $<50$ & $<50$ & $<50$ \\
\hline Hydrogen Sulfide & $\mathrm{mg} / \mathrm{kg}$ & $<50$ & $<50$ & $<50$ & $<50$ \\
\hline Oil and Grease & $\mathrm{mg} / \mathrm{L}$ & $<5.4$ & $<5.3$ & $<5.0$ & $<5.3$ \\
\hline Total Organic Carbon (TOC) & $\mathrm{mg} / \mathrm{L}$ & $<10$ & No data & $<10$ & 18 \\
\hline $\begin{array}{l}\text { Total Organic Halides } \\
\text { (TOX) }\end{array}$ & $\mu \mathrm{g} / \mathrm{L}$ & 13.6 & $<10$ & 12.3 & $<10$ \\
\hline \multicolumn{6}{|c|}{ Radionuclides } \\
\hline Americium-241 & $\mathrm{pCi} / \mathrm{L}$ & $-0.0057 \pm 0.36$ & $0.10 \pm 0.30$ & $-0.04 \pm 0.25$ & $0.05 \pm 0.26$ \\
\hline Curium-243/244 & $\mathrm{pCi} / \mathrm{L}$ & $-0.013 \pm 0.20$ & $-0.016 \pm 0.094$ & $0.01 \pm 0.25$ & $0.05 \pm 0.23$ \\
\hline Neptunium-237 & $\mathrm{pCi} / \mathrm{L}$ & $0.17 \pm 0.39$ & $0.01 \pm 0.13$ & $0.05 \pm 0.26$ & $0.09 \pm 0.24$ \\
\hline Plutonium-238 & $\mathrm{pCi} / \mathrm{L}$ & $0.05 \pm 0.29$ & $-0.09 \pm 0.18$ & $-0.03 \pm 0.18$ & $0.01 \pm 0.24$ \\
\hline Plutonium-239/240 & $\mathrm{pCi} / \mathrm{L}$ & $0.02 \dot{3} \pm 0.090$ & $-0.02 \pm 0.13$ & $0.022 \pm 0.085$ & $0.02 \pm 0.17$ \\
\hline Thorium-228 & $\mathrm{pCi} / \mathrm{L}$ & $0.21 \pm 0.43$ & $0.10 \pm 0.43$ & $0.13 \pm 0.35$ & $0.70 \pm 0.63$ \\
\hline Thorium-230 & $\mathrm{pCi} / \mathrm{L}$ & $0.59 \pm 0.57$ & $0.89 \pm 0.74$ & $0.11 \pm 0.27$ & $0.22 \pm 0.35$ \\
\hline Thorium-232 & $\mathrm{pCi} / \mathrm{L}$ & $0.01 \pm 0.16$ & $0.07 \pm 0.18$ & $0.01 \pm 0.15$ & $-0.01 \pm 0.17$ \\
\hline Uranium-234 & $\mathrm{pCi} / \mathrm{L}$ & $0.11 \pm 0.22$ & $0.07 \pm 0.17$ & $0.17 \pm 0.30$ & $0.19 \pm 0.35$ \\
\hline Uranium-235 & $\mathrm{pCi} / \mathrm{L}$ & $0 \pm 0.027$ & $0 \pm 0.027$ & $0.05 \pm 0.25$ & $-0.07 \pm 0.17$ \\
\hline Uranium-238 & $\mathrm{pCi} / \mathrm{L}$ & $0.064 \pm 0.15$ & $0.13 \pm 0.25$ & $0.30 \pm 0.39$ & $0.31 \pm 0.37$ \\
\hline Carbon-14 & $\mathrm{pCi} / \mathrm{L}$ & $6510 \pm 6800$ & $2960 \pm 6700$ & $2410 \pm 6700$ & $2870 \pm 6700$ \\
\hline Cesium-137 & $\mathrm{pCi} / \mathrm{L}$ & $-0.26 \pm 2.4$ & $-0.076 \pm 2.3$ & $-0.86 \pm 2.6$ & $1.8 \pm 2.4$ \\
\hline
\end{tabular}


Table 2 (continued)

\begin{tabular}{lccccc}
\hline Analyte & Unit $^{a}$ & SIOUFB $^{b}$ & SIOUFB2 $^{c}$ & SIOU39ERB1 $^{d}$ & SIOU40ERB $^{e}$ \\
\hline Cobalt-60 & $\mathrm{pCi} / \mathrm{L}$ & $-0.45 \pm 2.1$ & $1.2 \pm 2.4$ & $3.2 \pm 3.3$ & $1.1 \pm 2.4$ \\
Alpha activity & $\mathrm{pCi} / \mathrm{L}$ & $-1.3 \pm 1.5$ & $0.6 \pm 2.3$ & $0.3 \pm 1.2$ & $0.3 \pm 2.6$ \\
Beta activity & $\mathrm{pCi} / \mathrm{L}$ & $-4.5 \pm 6.1$ & $0.4 \pm 7.3$ & $3.4 \pm 7.1$ & $2.8 \pm 6.7$ \\
Technetium-99 & $\mathrm{pCi} / \mathrm{L}$ & $-1 \pm 15$ & $6 \pm 15$ & $6 \pm 15$ & $3 \pm 15$ \\
Strontium-89/90 & $\mathrm{pCi} / \mathrm{L}$ & $2 \pm 10$ & $< \pm 9.8$ & $-1 \pm 10$ & $-1 \pm 10$ \\
Tritium & $\mathrm{pCi} / \mathrm{ml}$ & $<45$ & $<46$ & $<46$ \\
\hline
\end{tabular}

${ }^{a}$ Conversion factors: $\mathrm{mg} / \mathrm{L}=\mathrm{ppm}, \mu \mathrm{g} / \mathrm{L}=\mathrm{ppb}, \mathrm{mg} / \mathrm{kg}=\mathrm{ppm}, \mu \mathrm{g} / \mathrm{kg}=\mathrm{ppb}$.

SIOUFB1 corresponds to Lab Sample ID A973380012 and Chain of Custody Numbers 103955 and 103956.

cSIOUFB2 corresponds to Lab Sample ID A973530034 and Chain of Custody Number 103966.

${ }^{d}$ SIOU39ERB 1 corresponds to Lab Sample ID A973490043 and Chain of Custody Number 103961.

${ }^{e}$ SIOU40ERB1 corresponds to Lab Sample ID A973510115 and Chain of Custody Number 103964. 
Table 3. Results of analysis of sediment samples taken from impoundment 3540

\begin{tabular}{|c|c|c|c|c|}
\hline Analyte & Unit $^{a}$ & SIOU40COMPI ${ }^{b}$ & SIOU40WEST ${ }^{c}$ & SIOU40EAST ${ }^{d}$ \\
\hline \multicolumn{5}{|c|}{ TCLP for: Semi-Volatiles, Metals, Pesticides, Herbicides } \\
\hline Antimony & $\mathrm{mg} / \mathrm{L}$ & $<0.40$ & $<0.44$ & $<0.40$ \\
\hline Arsenic & $\mathrm{mg} / \mathrm{L}$ & $<0.40$ & $<0.40$ & $<0.40$ \\
\hline Barium & $\mathrm{mg} / \mathrm{L}$ & 1.4 & 1.4 & $<1.0$ \\
\hline Beryllium & $\mathrm{mg} / \mathrm{L}$ & $<0.0020$ & 0.0030 & $<0.0020$ \\
\hline Cadmium & $\mathrm{mg} / \mathrm{L}$ & 0.038 & 0.17 & 0.081 \\
\hline Chromium & $\mathrm{mg} / \mathrm{L}$ & $<0.040$ & $<0.040$ & $<0.040$ \\
\hline Copper & $\mathrm{mg} / \mathrm{L}$ & $<0.040$ & 2.3 & 0.89 \\
\hline Lead & $\mathrm{mg} / \mathrm{L}$ & 1.4 & 4.6 & 2.6 \\
\hline Nickel & $\mathrm{mg} / \mathrm{L}$ & 0.49 & 0.44 & 0.39 \\
\hline Silver & $\mathrm{mg} / \mathrm{L}$ & $<0.040$ & $<0.040$ & $<0.040$ \\
\hline Vanadium & $\mathrm{mg} / \mathrm{L}$ & $<0.040$ & $<0.040$ & $<0.040$ \\
\hline Zinc & $\mathrm{mg} / \mathrm{L}$ & 3.8 & 7.0 & 4.2 \\
\hline Mercury & $\mu \mathrm{g} / \mathrm{L}$ & $<20$ & $<20$ & $<20$ \\
\hline Selenium & $\mu \mathrm{g} / \mathrm{L}$ & $<20$ & $<20$ & $<20$ \\
\hline $2,4-D$ & $\mu \mathrm{g} / \mathrm{L}$ & $<4$ & $<4$ & $<4$ \\
\hline Silvex & $\mu \mathrm{g} / \mathrm{L}$ & $<4$ & $<4$ & $<4$ \\
\hline Chlordane & $\mu \mathrm{g} / \mathrm{L}$ & $<13$ & $<13$ & $<13$ \\
\hline Endrin & $\mu \mathrm{g} / \mathrm{L}$ & $<1.6$ & $<1.6$ & $<1.6$ \\
\hline Heptachlor & $\mu \mathrm{g} / \mathrm{L}$ & $<0.8$ & $<0.8$ & $<0.8$ \\
\hline Heptachlor epoxide & $\mu \mathrm{g} / \mathrm{L}$ & $<0.8$ & $<0.8$ & $<0.8$ \\
\hline Lindane & $\mu \mathrm{g} / \mathrm{L}$ & $<0.8$ & $<0.8$ & $<0.8$ \\
\hline
\end{tabular}


Table 3 (continued)

\begin{tabular}{|c|c|c|c|c|}
\hline Analyte & Unit $^{a}$ & SIOU $40 C O M P 1^{b}$ & SIOU $40 W E S T^{c}$ & ${\text { SIOU } 40 \mathrm{EAST}^{d}}^{d}$ \\
\hline Methoxychlor & $\mu \mathrm{g} / \mathrm{L}$ & $<8$ & $<8$ & $<8$ \\
\hline Toxaphene & $\mu \mathrm{g} / \mathrm{L}$ & $<13$ & $<13$ & $<13$ \\
\hline 1,4-Dichlorobenzene & $\mu \mathrm{g} / \mathrm{kg}$ & $<10$ & $<10$ & $<10$ \\
\hline 2,4,5-Trichlorophenol & $\mu \mathrm{g} / \mathrm{kg}$ & $<10$ & $<10$ & $<10$ \\
\hline 2,4,6-Trichlorophenol & $\mu \mathrm{g} / \mathrm{kg}$ & $<10$ & $<10$ & $<10$ \\
\hline 2,4-Dinitrotoluene & $\mu \mathrm{g} / \mathrm{kg}$ & $<10$ & $<10$ & $<10$ \\
\hline 2-Methylphenol & $\mu \mathrm{g} / \mathrm{kg}$ & $<10$ & $<10$ & $<10$ \\
\hline 3- and 4- Methylphenol & $\mu \mathrm{g} / \mathrm{kg}$ & $<10$ & $<10$ & $<10$ \\
\hline Hexachlorobenzene & $\mu \mathrm{g} / \mathrm{kg}$ & $<10$ & $<10$ & $<10$ \\
\hline Hexachlorobutadiene & $\mu \mathrm{g} / \mathrm{kg}$ & $<10$ & $<10$ & $<10$ \\
\hline Hexachloroethane & $\mu \mathrm{g} / \mathrm{kg}$ & $<10$ & $<10$ & $<10$ \\
\hline Nitrobenzene & $\mu \mathrm{g} / \mathrm{kg}$ & $<10$ & $<10$ & $<10$ \\
\hline Pentachlorophenol & $\mu \mathrm{g} / \mathrm{kg}$ & $<10$ & $<10$ & $<10$ \\
\hline Pyridine & $\mu \mathrm{g} / \mathrm{kg}$ & $<10$ & $<20$ & $<20$ \\
\hline \multicolumn{5}{|c|}{ PCBs (Total and Aroclor-Specific) } \\
\hline Aroclor-1016 & $\mu \mathrm{g} / \mathrm{kg}$ & $<2000$ & $<1500$ & $<1500$ \\
\hline Aroclor-1221 & $\mu \mathrm{g} / \mathrm{kg}$ & $<2000$ & $<1500$ & $<1500$ \\
\hline Aroclor- 1232 & $\mu \dot{\mathrm{g}} / \mathrm{kg}$ & $<2000$ & $<1500$ & $<1500$ \\
\hline Aroclor- 1242 & $\mu \mathrm{g} / \mathrm{kg}$ & $<2000$ & $<1500$ & $<1500$ \\
\hline Aroclor- 1248 & $\mu \mathrm{g} / \mathrm{kg}$ & $<2000$ & $<1500$ & $<1500$ \\
\hline Aroclor-1254 & $\mu \mathrm{g} / \mathrm{kg}$ & 4600 & 8000 & 2700 \\
\hline Aroclor- 1260 & $\mu \mathrm{g} / \mathrm{kg}$ & 2000 & 1500 & 1500 \\
\hline
\end{tabular}




\section{Table 3 (continued)}

\begin{tabular}{|c|c|c|c|c|}
\hline Analyte & Unit $^{a}$ & SIOU40COMPI ${ }^{b}$ & SIOU40WEST $^{c}$ & 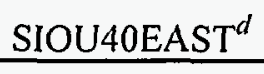 \\
\hline PCB, Total & $\mu \mathrm{g} / \mathrm{kg}$ & 4600 & 8000 & 2700 \\
\hline \multicolumn{5}{|c|}{ Reactive Cyanide, Reactive Sulfide, Oil and Grease, Total Organic Halogen (TOX) } \\
\hline Cyanide & $\mathrm{mg} / \mathrm{kg}$ & $<50$ & $<50$ & $<50$ \\
\hline Hydrogen Sulfide & $\mathrm{mg} / \mathrm{kg}$ & 120 & 70 & 50 \\
\hline Oil and Grease & $\mu \mathrm{g} / \mathrm{g}$ & 2100 & 1700 & 280 \\
\hline $\begin{array}{l}\text { Total Organic Halides } \\
\text { (TOX) }\end{array}$ & $\mu \mathrm{g} / \mathrm{g}$ & 25 & $<25$ & 30 \\
\hline \multicolumn{5}{|c|}{ Radionuclides (dry weight basis) } \\
\hline Americium-241 & $\mathrm{pCi} / \mathrm{g}$ & $2.9 \pm 0.44$ & $1.4 \pm 0.19$ & $2.0 \pm 0.22$ \\
\hline Curium-243/244 & $\mathrm{pCi} / \mathrm{g}$ & $19 \pm 1.8$ & $12 \pm 0.92$ & $18 \pm 1.3$ \\
\hline Neptunium-237 & $\mathrm{pCi} / \mathrm{g}$ & $0.091 \pm 0.084$ & $0.017 \pm 0.036$ & $0.014 \pm 0.034$ \\
\hline Plutonium-238 & $\mathrm{pCi} / \mathrm{g}$ & $4.9 \pm 1$ & $3.3 \pm 0.7$ & $3.9 \pm 0.8$ \\
\hline Plutonium-239/240 & $\mathrm{pCi} / \mathrm{g}$ & $2.9 \pm 0.6$ & $2.4 \pm 0.5$ & $5.1 \pm 1$ \\
\hline Thorium-228 & $\mathrm{pCi} / \mathrm{g}$ & $16 \pm 4$ & $12 \pm 4$ & $13 \pm 3$ \\
\hline Thorium-230 & $\mathrm{pCi} / \mathrm{g}$ & $1.8 \pm 0.5$ & $1.2 \pm 1$ & $1.3 \pm 0.4$ \\
\hline Thorium-232 & $\mathrm{pCi} / \mathrm{g}$ & $10 \pm 3$ & $11 \pm 4$ & $9.4 \pm 2.3$ \\
\hline Uranium-233 $3^{\circ}$ & $\mathrm{pCi} / \mathrm{g}$ & 1 & 2 & 1 \\
\hline Uranium-234 & $\mathrm{pCi} / \mathrm{g}$ & $19 \pm 4$ & $14 \pm 3$ & $22 \pm 4$ \\
\hline Uranium-235 & $\mathrm{pCi} / \mathrm{g}$ & $0.66 \pm 0.22$ & $0.48 \pm 0.18$ & $0.50 \pm 0.19$ \\
\hline Uranium-238 & $\mathrm{pCi} / \mathrm{g}$ & $9.4 \pm 1.8$ & $7.2 \pm 1.4$ & $8.0 \pm 1.6$ \\
\hline Carbon-14 & $\mathrm{pCi} / \mathrm{g}$ & $19.2 \pm 21$ & $26.2 \pm 22$ & $22.9 \pm 21$ \\
\hline Cesium-137 & $\mathrm{pCi} / \mathrm{g}$ & $78 \pm 6.1$ & $63 \pm 7.5$ & $62 \pm 6.1$ \\
\hline Cobalt- 60 & $\mathrm{pCi} / \mathrm{g}$ & $0.89 \pm 0.15$ & $0.94 \pm 0.15$ & $1.2 \pm 0.15$ \\
\hline
\end{tabular}


Table 3 (continued)

\begin{tabular}{lcccc}
\hline Analyte & Unit $^{a}$ & SIOU40COMP1 $^{b}$ & SIOU40WEST $^{c}$ & SIOU40EAST $^{d}$ \\
\hline Europium-152 & $\mathrm{pCi} / \mathrm{g}$ & $13 \pm 0.90$ & $10 \pm 0.74$ & $11 \pm 0.84$ \\
Europium-154 & $\mathrm{pCi} / \mathrm{g}$ & $4.1 \pm 0.73$ & $2.7 \pm 0.29$ & $3.0 \pm 0.53$ \\
Europium-155 & $\mathrm{pCi} / \mathrm{g}$ & $2.1 \pm 0.71$ & $4.0 \pm 0.77$ & $1.5 \pm 0.64$ \\
Osmium-191 & $\mathrm{pCi} / \mathrm{g}$ & $2.7 \pm 1.0$ & $2.6 \pm 0.66$ & $1.3 \pm 0.66$ \\
Potassium-40 & $\mathrm{pCi} / \mathrm{g}$ & $10 \pm 3.0$ & $6.8 \pm 2.0$ & $7.7 \pm 2.0$ \\
Alpha activity & $\mathrm{pCi} / \mathrm{g}$ & $110 \pm 10$ & $66 \pm 10$ & $100 \pm 10$ \\
Beta activity & $\mathrm{pCi} / \mathrm{g}$ & $270 \pm 20$ & $160 \pm 10$ & $210 \pm 10$ \\
Technetium-99 & $\mathrm{pCi} / \mathrm{g}$ & $17 \pm 2$ & $10 \pm 2$ & $9.2 \pm 0.6$ \\
Strontium-90 & $\mathrm{pCi} / \mathrm{g}$ & $67 \pm 2$ & $38 \pm 2$ & $48 \pm 2$ \\
Tritium & $\mathrm{pCi} / \mathrm{g}$ & $<142$ & $<149$ & $<146$ \\
\hline
\end{tabular}

${ }^{a}$ Conversion factors: $\mathrm{mg} / \mathrm{L}=\mathrm{ppm}, \mu \mathrm{g} / \mathrm{L}=\mathrm{ppb}, \mu \mathrm{g} / \mathrm{g}=\mathrm{ppm}, \mathrm{mg} / \mathrm{kg}=\mathrm{ppm}, \mu \mathrm{g} / \mathrm{kg}=\mathrm{ppb}$.

${ }^{b}$ SIOU40COMP1 corresponds to Lab Sample ID A973490068 and Chain of Custody Number 103962.

${ }^{c}$ SIOU40WEST corresponds to Lab Sample ID A973520166 and Chain of Custody Number 103965.

${ }^{d}$ SIOU40EAST corresponds to Lab Sample ID A973510116 and Chain of Custody Number 103963.

${ }^{e}$ Uranium-233 values are estimated based on visual observations of the spectra by the authors. ASO documentation qualifies U-233 present but does not quantify any values. 
Table 4. Results of particle-size test for sediment samples from impoundments 3539 and 3540

\begin{tabular}{lcccc}
\hline & \multicolumn{3}{c}{ Diameter $(\mu \mathrm{m})$} \\
\cline { 2 - 4 } Project Sample ID & $\begin{array}{c}10 \% \\
\text { Passthrough }\end{array}$ & $100 \%$ Passthrough & $50 \%$ Passthrough & $\begin{array}{c}90 \% \\
\text { Passthrough }\end{array}$ \\
\hline SIOU39COMP1 & 2.94 & 176 & 13.93 & 62.44 \\
SIOU39WEST & 2.87 & 125 & 8.26 & 45.89 \\
SIOU39EAST & 2.55 & 125 & 8.69 & 56.23 \\
SIOU39DUP1 & 2.92 & 176 & 14.80 & 61.08 \\
SIOU40COMP1 & 3.22 & 176 & 17.71 & 56.93 \\
SIOU40WEST & 2.86 & 176 & 8.49 & 50.73 \\
SIOU40EAST & 2.82 & 125 & 10.97 & 54.34 \\
\hline
\end{tabular}




\title{
APPENDIX A \\ SAMPLING AND ANALYSIS PLAN \\ SIOU Impoundments 3539 and 3540 Sampling Project
}

\author{
Date Issued - March 11, 1998 \\ Revision 3 \\ Prepared by: \\ ORNL / MAD \\ Measurement Applications and Development Group \\ Life Sciences Division \\ Oak Ridge National Laboratory
}




\section{SAMPLING AND ANALYSIS PLAN APPROVALS AND CONCURRENCES}

(Original signed and located in project files)

\section{APPROVALS:}

R. A. Dean

Date:

Project Manager

M. E. Murray

Date:

MAD Project Manager

Date:

T. M. Koepp

Quality Assurance Representative

J. D. Kopotic

DOE Representative

\section{Date:}

\section{CONCURRENCES:}

D. A. Rose / D. E. Rice

Date:

Site Health and Safety Officers

D. A. Rose

MAD Field Team Leader

Date: 


\section{TABLE OF CONTENTS}

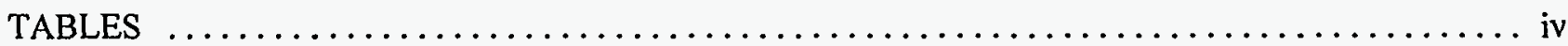

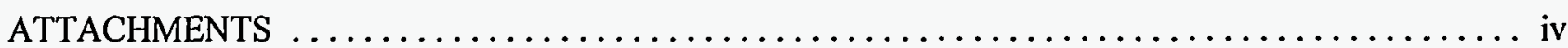

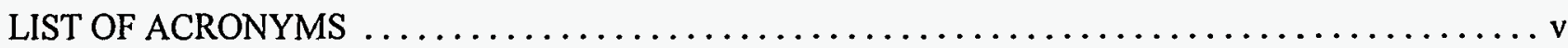

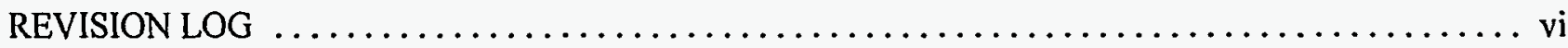

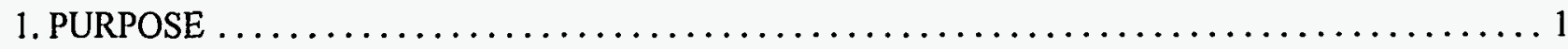

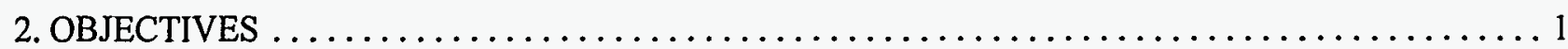

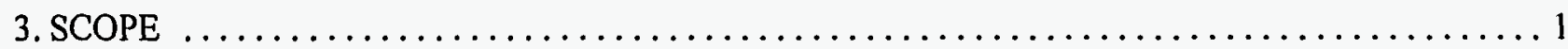

4. ORGANIZATION AND RESPONSIBILITIES $\ldots \ldots \ldots \ldots \ldots \ldots \ldots \ldots \ldots \ldots \ldots \ldots \ldots \ldots \ldots \ldots \ldots$

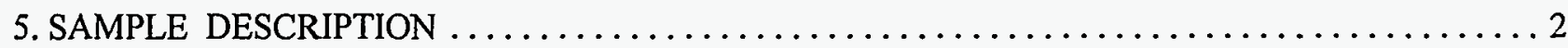

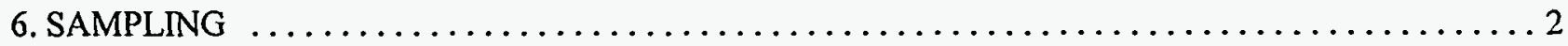

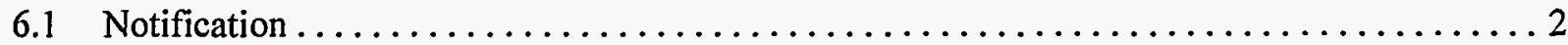

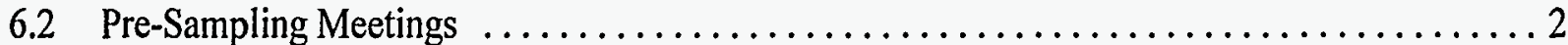

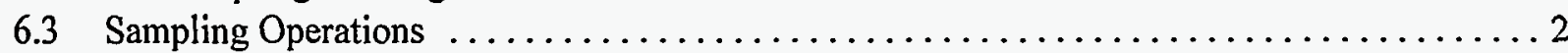

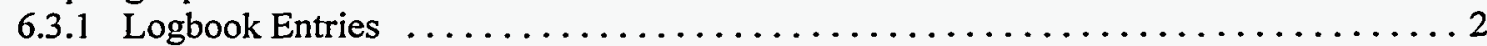

6.3.2 Sample Collection Overview $\ldots \ldots \ldots \ldots \ldots \ldots \ldots \ldots \ldots \ldots \ldots \ldots \ldots \ldots \ldots \ldots \ldots \ldots$

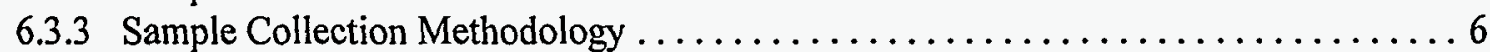

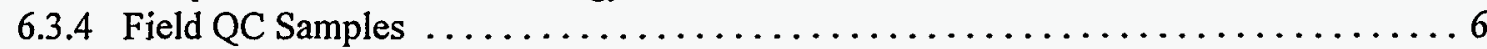

6.3.5 Sampling Equipment Decontamination $\ldots \ldots \ldots \ldots \ldots \ldots \ldots \ldots \ldots \ldots \ldots \ldots \ldots \ldots \ldots \ldots$

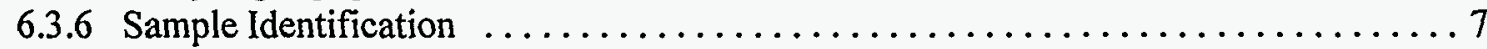

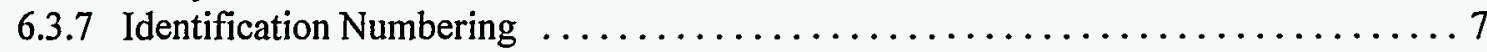

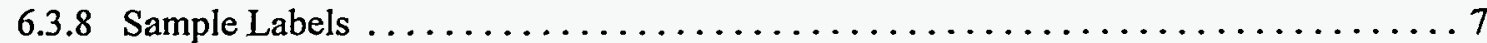

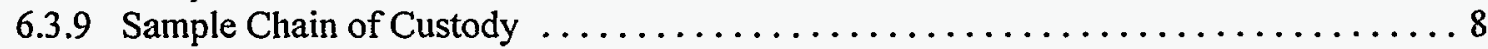

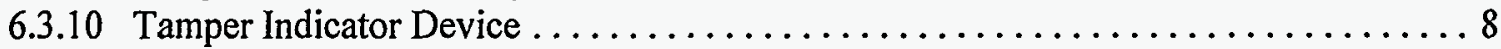

6.3 .11 Sample Management $\ldots \ldots \ldots \ldots \ldots \ldots \ldots \ldots \ldots \ldots \ldots \ldots \ldots \ldots \ldots \ldots$

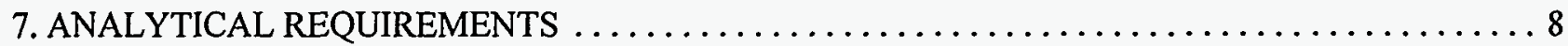

8. QUALITY ASSURANCE (QA) REQUIREMENTS $\ldots \ldots \ldots \ldots \ldots \ldots \ldots \ldots \ldots \ldots \ldots \ldots$

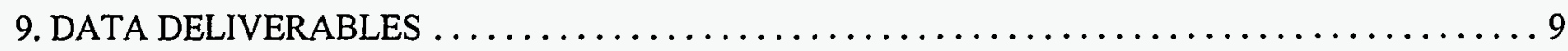

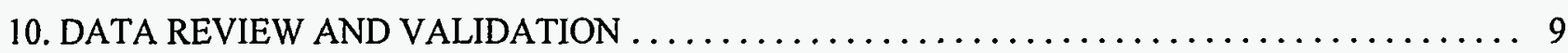

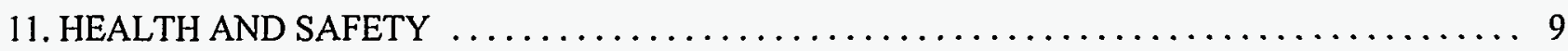

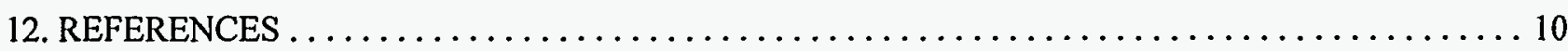




\section{TABLES}

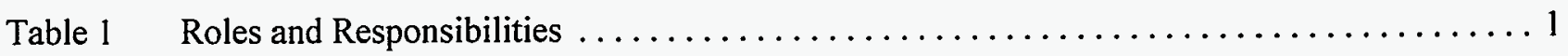

Table 2 Requirements for Sediment/Sludge Samples $\ldots \ldots \ldots \ldots \ldots \ldots \ldots \ldots \ldots \ldots \ldots \ldots$

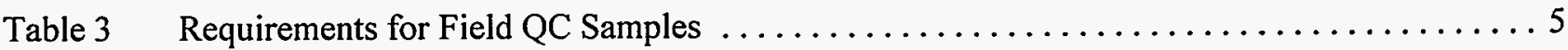

\section{ATTACHMENTS}

Attachment A Sampling Locations for Pond $3539 \ldots \ldots \ldots \ldots \ldots \ldots \ldots \ldots \ldots \ldots \ldots \ldots \ldots$

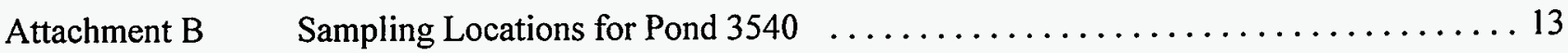

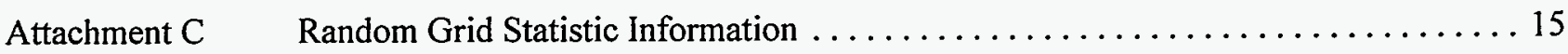




\section{LIST OF ACRONYMS}

$\begin{array}{ll}\text { AMS } & \text { American Meteorological Society } \\ \text { EPA } & \text { Environmental Protection Agency } \\ \text { ER } & \text { Environmental Restoration } \\ \text { ERB } & \text { Equipment Rinsate Blank } \\ \text { SHEST } & \text { Safety \& Health Evaluation Support Team } \\ \text { LMER } & \text { Lockheed Martin Energy Research } \\ \text { LMES } & \text { Lockheed Martin Energy Systems } \\ \text { MAD } & \text { Measurement Applications and Development Group } \\ \text { ORNL } & \text { Oak Ridge National Laboratory } \\ \text { PCB } & \text { Polychlorinated Biphenyl } \\ \text { QA/QC } & \text { Quality Assurance/Quality Control } \\ \text { RCRA } & \text { Resource Conservation and Recovery Act } \\ \text { S\&A } & \text { Sampling and Analysis } \\ \text { SAP } & \text { Sampling and Analysis Plan } \\ \text { SIOU } & \text { Surface Impoundment Operable Unit } \\ \text { TCLP } & \text { Toxicity Characteristic Leaching Procedure } \\ \text { TID } & \text { Tamper Indicator Device } \\ \text { TSCA } & \text { Toxic Substances Control Act }\end{array}$




\section{REVISION LOG}

\section{Revision No.}

$0 \quad 11-5-97$

$1 \quad 11-26-97$

2

$12-9-97$

3

$2-20-98$

\section{Description of Changes}

New Document

P.1; Changed "SMO" to "Analytical" \& "Christy Anderson" to "Angela Barnard-Hatmaker"

Corrected Tables $2 \& 3$ Analytical Methods to reflect proper methods for sediment matrix

Included typed names on the sign off page Added REVISION LOG

Changed Tables $2 \& 3$ sample volumes per ASO recommendations.

Section 6.3.2, Added systematic composite sample requirements and deleted requirements for field duplicates.

Modifications resulting from QA Surveillance (97SIOU-1).

This revision is for documentation purposes only, sampling and analyses are complete. The following sections were modified: 4.0 Table $1,6.2,6.3 .1,6.3 .2$, 6.3.3, and 6.3.5. 


\section{Sampling and Analysis Plan for SIOU Impoundments 3539 and 3540 Sampling Project}

\section{PURPOSE}

This Sampling and Analysis Plan (SAP) describes the methodology to obtain further characterization information on the sediment/clay materials found in the SIOU Impoundments 3539 and 3540, located at Oak Ridge National Laboratory (ORNL). Characterization data obtained from the representative sampling of the sediment/clay materials will be used to evaluate the impoundment closure conditions.

\section{OBJECTIVES}

The objective of this SAP is to describe the procedure for obtaining sufficient and valid analytical data on sediment/clay materials to evaluate RCRA and/or TSCA regulatory applicability. Additionally, analytical data will be obtained to evaluate radiological and sediment/clay physical properties. This will provide information for subsequent treatment, storage, packaging, and shipment to off-site facilities.

\section{SCOPE}

This plan provides supplemental instructions to guidelines and procedures established for Sampling and Analysis (S\&A) activities. Standard procedures may be referenced throughout this plan as applicable, and are available for review if necessary.

\section{ORGANIZATION AND RESPONSIBILITIES}

Overall coordination and implementation of the S\&A activities described in this plan are the responsibility of the MAD Field Team Leader. The MAD Field Team Leader will require input and support from personnel of various ORNL organizations. The roles and responsibilities of these personnel are listed in Table 1.

\begin{tabular}{|l|l|c|l|}
\hline \multicolumn{1}{|c|}{ Role } & \multicolumn{1}{|c|}{ Person } & Phone & \multicolumn{1}{c|}{ Responsibility } \\
\hline $\begin{array}{l}\text { MAD Field } \\
\text { Team Leader }\end{array}$ & Doug Rose & $574-5837$ & $\begin{array}{l}\text { Oversees all activities involving sampling, maintains field } \\
\text { logbook, access to the work areas, and support of outside } \\
\text { organizations }\end{array}$ \\
\hline $\begin{array}{l}\text { SHEST } \\
\text { Coordinator }\end{array}$ & Greg Rowland & $576-6445$ & $\begin{array}{l}\text { Coordinates all aspects of the characterization project } \\
\text { relating to SHEST support }\end{array}$ \\
\hline $\begin{array}{l}\text { Quality } \\
\text { Assurance }\end{array}$ & Tom Koepp & $576-8057$ & $\begin{array}{l}\text { Coordinates QA support and ensures QA compliance for the } \\
\text { project }\end{array}$ \\
\hline $\begin{array}{l}\text { Analytical } \\
\text { Coordinator }\end{array}$ & Angela Barnard- & $241-9669$ & $\begin{array}{l}\text { Ensures the requirements of the SAP are met by the } \\
\text { laboratories and provides customer interface }\end{array}$ \\
\hline $\begin{array}{l}\text { Project } \\
\text { Manager }\end{array}$ & Robert Dean & $574-7951$ & Oversees project management and sampling activities \\
\hline $\begin{array}{l}\text { Project } \\
\text { Manager }\end{array}$ & Rob Coe & $241-5026$ & Provides engineering support for the project \\
\hline $\begin{array}{l}\text { MAD Project } \\
\text { Manager }\end{array}$ & Michael Murray & $574-5838$ & $\begin{array}{l}\text { Oversees all MAD group activities, interfaces with project } \\
\text { managers, ensures proper training and documentation. }\end{array}$ \\
\hline $\begin{array}{l}\text { Health and } \\
\text { Safety Officer }\end{array}$ & Dennis Rice & $576-8565$ & $\begin{array}{l}\text { Provides health and safety oversight and assists in sampling } \\
\text { activities }\end{array}$ \\
\hline
\end{tabular}




\section{SAMPLE DESCRIPTION}

The sediment/clay identified for characterization using this SAP is currently contained on the bottom of SIOU Impoundments 3539 and 3540 . The impoundments were used as part of the system for management of low-level radioactive wastes generated from experiments and material processing at ORNL. The impoundments contain $\sim 40$ cubic yards of sediment which include organic materials. The media constituents will contain organic materials such as leaves, small twigs, decomposed organic materials, and about 0.1 foot of the clay liner. The various sediment depths have been estimated using earlier collected data.

\section{SAMPLING}

\subsection{NOTIFICATION}

The MAD Field Team Leader will notify the analytical organization and the project management before sampling activities begin. This notification is needed to allow the Analytical Coordinator time to schedule programmatic activities. The Analytical Coordinator shall notify laboratory analysis personnel regarding the time and date of sampling operations.

\subsection{PRE-SAMPLING MEETINGS}

The MAD Project Manager will schedule and conduct a pre-sampling meeting with project personnel prior to sampling operations. The objectives of this meeting will be to discuss sampling operation logistics and other details, resolve any technical or operational issues, and ensure schedules are agreed upon by all responsible organizations. The Project Manager will also verify training records pertinent to this project are complete and available for inspection.

\subsection{SAMPLING OPERATIONS}

The MAD Field Team Leader will ensure sampling activities adhere to the SAP and the Health and Safety Plan, and applicable LMER and LMES procedures. Sampling shall not be initiated prior to receipt of a signed copy of the SAP. Signed copies of the SAP will be distributed prior to the initiation of sampling activities.

\subsubsection{Logbook Entries}

The Field Team leader (or designee) will maintain a logbook to provide project information and a daily written record of all sampling activities. The logbook will be maintained in accordance with Quality Assurance Plan for the Oak Ridge National Laboratory Environmental Restoration Program, ORNL/ER-225/R1. Project information should include personnel contacts, trainings activities and site data. Each daily logbook entry should include, but is not limited to, the following items:

- sediment/sludge/sample description (e.g., color, texture, solids content);

- sample container IDs;

- approximate amount of material in each sample container;

- customer sample numbers;

- personnel; and

- chain-of-custody number.

\subsubsection{Sample Collection Overview}

Sampling activities will require collection of three types of analytical samples. The samples will consist of composite random samples, composite systematic, and field quality control (QC) samples. Sampling will involve intrusive and random methods. The sampling team will follow the general guidelines of the LMES procedure Collection of Sediment Samples, ESP-304-1, Rev. 1, dated February 28, 1996. Location samples will be collected and contained in appropriate sample containers (specified in Table 2). In all sampling instances, a one-tenth foot clay (liner) portion will be included with the sediment/sludge. The one-tenth foot section of the clay (liner) will be homogenized as part of the sample. 
Four composite samples will be collected from each pond. Each composite sample will consist of sampled media from 18 randomly selected locations. The sample locations will be selected from a grid system generated by MAD personnel. The grid system will be designated as 2 feet $x 2$ feet and will lie on an X-Y axis relative to the ORNL grid north direction from the impoundments (see Attachments $A$ and $B$ ). The number of samples collected should represent the media of each impoundment. At the direction of the DOE Project manager and the LMER Project manager, the number of composite samples may be altered based on changing project goals and/or analytical data as it becomes available.

Seventy-two (72) sampling locations will be selected from each impoundment for active sampling activities. From these seventy-two locations, eighteen numerically labeled locations will be selected for each of the four (4) composite samples to be collected in each impoundment (i.e., the first eighteen locations labeled with \#1 will comprise the \#1 composite sample). Alternative locations will be generated during the random grid selection for intrusion refusal at initial locations. The statistical basis for this sampling regime was provided by Jacobs Engineering (see Attachment C). The Field Team Leader will make the decision for sample collections at each location. Conditions such as insufficient sediment/sludge, etc., will determine the location changes to be made, if necessary.

The systematic composite samples will consist of sediment collected from 9 grid point locations on the west side of each pond and a similar composite from the east side of each pond. A grid will be established to designate 18 distinct sampling blocks ( 9 per east/west side). A sediment/clay sample will be collected from each of the 9 blocks and then added to the composite.

Specific equipment for taking samples may include stainless collection devices (Shelby tubes, stainless steel trays, AMS coring devices, etc.), a low-volume pump and related equipment for removing excess liquid from the sample locations, and a modified stainless steel drum for creating a seal and containing the sediment/sludge in the selected sample location. Other supplies and equipment will include standard sample bottles, sample handling tools such as stainless steel scoops and spatulas, and radiological contamination control supplies. Any deviations from this method will be approved by the MAD Field Team Leader, project management, and/or the Analytical Coordinator. Equipment that is fabricated and designed on-site must meet the requirements for analytical standards, and support the needs of the sampling team. The equipment should be designed with decontamination processes in mind, and should be fabricated from materials meeting analysis criteria. 


\begin{tabular}{|c|c|c|c|c|}
\hline Analyte & Analytical Methods & $\begin{array}{l}\text { Container } \\
\text { Type }\end{array}$ & Preservative & $\begin{array}{l}\text { Sample } \\
\text { Volumes }\end{array}$ \\
\hline $\begin{array}{l}\text { TCLP for: Semi- } \\
\text { volatiles, metals } \\
\text { (including } \mathrm{Hg}, \mathrm{Cu} \text {, } \\
\text { and } \mathrm{Zn} \text { ), pesticides, } \\
\text { herbicides }\end{array}$ & $\begin{array}{l}\text { SW-846, } 1311 \\
\text { UTS } 6010,7421, \\
7470,7740,8080\end{array}$ & $\begin{array}{l}1 \text { liter, amber, with } \\
\text { Teflon-lined lid }\end{array}$ & 4 degrees $C$ & $\begin{array}{l}1 \text { liter (Fill } \\
\text { sample jar } \\
\text { completely) }\end{array}$ \\
\hline $\begin{array}{l}\text { PCBs (total and } \\
\text { Aroclor-specific) }\end{array}$ & SW-846, 8081 & $\begin{array}{l}1 \text { liter, amber, with } \\
\text { Teflon-lined lid }\end{array}$ & 4 degrees $\mathrm{C}$ & 1 liter \\
\hline $\begin{array}{l}\text { Reactive cyanide, } \\
\text { Reactive sulfide }\end{array}$ & $\begin{array}{l}\text { SW-846, Methods } \\
7.3 .3 .2,7.3 .4 .2\end{array}$ & \multirow{4}{*}{$\begin{array}{l}1 \text { liter, amber, with } \\
\text { Teflon-lined lid }\end{array}$} & \multirow{4}{*}{4 degrees $\mathrm{C}$} & \multirow{4}{*}{$\begin{array}{l}1 \text { liter (Fill } \\
\text { sample jar } \\
\text { completely) }\end{array}$} \\
\hline Oil and Grease & $\begin{array}{l}\text { SW-846, Method } \\
9071 \mathrm{~A}\end{array}$ & & & \\
\hline $\begin{array}{l}\text { Total organic carbon } \\
\text { (TOC), total organic } \\
\text { halogen (TOX) }\end{array}$ & $\begin{array}{l}\text { EPA } 415.1 \text { (TOC) } \\
\text { SW-846, Method } \\
9020 B(T O X)\end{array}$ & & & \\
\hline Particle size analysis & ASTM D422 & & & \\
\hline $\begin{array}{l}\text { Radionuclides } \\
\text { Isotopic-Pu } \\
\text { Isotopic-U } \\
\text { Isotopic-Th } \\
\text { Technicium-99 } \\
\text { Americium-241 } \\
\text { Neptunium-237 } \\
\text { Gamma } \\
\text { Spectroscopy } \\
\text { Strontium-90 } \\
\text { Gross Alpha/Beta } \\
\text { Tritium } \\
\text { Carbon-14 }\end{array}$ & $\begin{array}{l}\text { Alpha Spectroscopy } \\
\text { Alpha Spectroscopy } \\
\text { Alpha Spectroscopy } \\
\text { Liquid Scintillation } \\
\text { Alpha Spectroscopy } \\
\text { Alpha Spectroscopy } \\
\text { Gamma Emitters } \\
\text { Beta GPC } \\
\text { Gross Alpha/Beta } \\
\text { Liquid Scintillation } \\
\text { Liquid Scintillation }\end{array}$ & $\begin{array}{l}1 \text { liter, amber, with } \\
\text { Teflon-lined lid }\end{array}$ & 4 degrees $C$ & 2 liters \\
\hline
\end{tabular}




\begin{tabular}{|c|c|c|c|c|c|}
\hline \multicolumn{6}{|c|}{ Table 3. Requirements for Field QC Samples } \\
\hline $\begin{array}{l}\text { Sample } \\
\text { Type }\end{array}$ & Analytes & Analytical Methods & Container Type & Preservative & $\begin{array}{l}\text { Sample } \\
\text { Volume }\end{array}$ \\
\hline $\begin{array}{l}\text { Field } \\
\text { Duplicate }\end{array}$ & Same as Table 2 & Same as Table 2 & $\begin{array}{l}\text { Same as } \\
\text { Table } 2\end{array}$ & $\begin{array}{l}\text { Same as } \\
\text { Table } 2\end{array}$ & $\begin{array}{l}\text { Same as } \\
\text { Table } 2\end{array}$ \\
\hline \multirow[t]{6}{*}{$\begin{array}{l}\text { Equipment } \\
\text { Rinsate } \\
\text { Blank }\end{array}$} & $\begin{array}{l}\text { TCLP for: Semi- } \\
\text { volatiles, metals } \\
\text { (including } \mathrm{Hg}, \mathrm{Cu} \text {, } \\
\text { and } \mathrm{Zn} \text { ), } \\
\text { pesticides, } \\
\text { herbicides }\end{array}$ & $\begin{array}{l}\text { SW-846, 6010A, } \\
7470\end{array}$ & $\begin{array}{l}1 \text { liter, amber, } \\
\text { w/teflon lined } \\
\text { lid }\end{array}$ & 4 degrees $C$ & $\begin{array}{l}3 \text { liter (Fill } \\
\text { sample jar } \\
\text { completely) }\end{array}$ \\
\hline & $\begin{array}{l}\text { PCBs (total and } \\
\text { Aroclor-specific) }\end{array}$ & SW-846, 8081 & $\begin{array}{l}1 \text { liter, amber, } \\
\text { w/teflon lined } \\
\text { lid }\end{array}$ & 4 degrees $\mathrm{C}$ & 1 liter \\
\hline & $\begin{array}{l}\text { Reactive cyanide, } \\
\text { Reactive sulfide }\end{array}$ & $\begin{array}{l}\text { SW-846, Methods } \\
7.3 .3 .2,7.3 .4 .2\end{array}$ & $\begin{array}{l}1 \text { liter, amber, } \\
\text { w/teflon lined } \\
\text { lid }\end{array}$ & 4 degrees $\mathrm{C}$ & 1 liter \\
\hline & Oil and Grease & $\begin{array}{l}\text { SW-846, Method } \\
413.2\end{array}$ & $\begin{array}{l}1 \text { liter, amber, } \\
\text { w/teflon lined } \\
\text { lid }\end{array}$ & 4 degrees $C$ & 1 liter \\
\hline & $\begin{array}{l}\text { Total organic } \\
\text { carbon (TOC), } \\
\text { total organic } \\
\text { halogen (TOX) }\end{array}$ & $\begin{array}{l}\text { EPA 415.1 (TOC) } \\
\text { SW-846, Method } \\
9020 B \text { (TOX) }\end{array}$ & $\begin{array}{l}250 \mathrm{ml} . \text {, amber, } \\
\text { w/teflon lined } \\
\text { lid, (1) } 250 \mathrm{ml} \\
\text { for TOC, (1) } 250 \\
\text { ml. for TOX }\end{array}$ & 4 degrees $\mathrm{C}$ & $\begin{array}{l}250 \mathrm{ml} \text {. (Fill } \\
\text { each } 250 \mathrm{ml} \text {. } \\
\text { Sample } \\
\text { bottle } \\
\text { completely) }\end{array}$ \\
\hline & $\begin{array}{l}\text { Radionuclides } \\
\text { Isotopic-Pu } \\
\text { Isotopic-U } \\
\text { Isotopic-Th } \\
\text { Technicium-99 } \\
\text { Americium-241 } \\
\text { Neptunium-237 } \\
\text { Gamma } \\
\text { Spectroscopy } \\
\text { Strontium-90 } \\
\text { Gross Alpha/Beta } \\
\text { Tritium } \\
\text { Carbon-14 }\end{array}$ & $\begin{array}{l}\text { Alpha Spectroscopy } \\
\text { Alpha Spectroscopy } \\
\text { Alpha Spectroscopy } \\
\text { Liquid Scintillation } \\
\text { Alpha Spectroscopy } \\
\text { Alpha Spectroscopy } \\
\text { Gamma Emitters } \\
\text { Beta GPC } \\
\text { Gross Alpha/Beta } \\
\text { Liquid Scintillation } \\
\text { Liquid Scintillation }\end{array}$ & $\begin{array}{l}1 \text { liter amber } \\
\text { w/teflon lined } \\
\text { lid }\end{array}$ & 4 degrees $\mathrm{C}$ & 2 liters \\
\hline Field Blank & Same as ERBs & Same as ERBs & Same as ERBs & Same as ERBs & $\begin{array}{l}\text { Same as } \\
\text { ERBs }\end{array}$ \\
\hline
\end{tabular}




\subsubsection{Sample Collection Methodology}

After the conclusion of the pre-sampling meeting, all materials necessary for sample collection will be staged and inspected prior to entering the radiological buffer area. A Field Blank (FB) sample and the Equipment Rinsate Blank (ERB) samples will be collected at this time for the deionized water and on all reusable sampling equipment (see Table 3 for field QC requirements). The selected sampling locations will then be accessed using a boat and/or walkboards strategically located across the impoundments.

After proceeding to the selected sample location, verify the coordinates and log the location. If the water level is high enough to be a problem for sampling tool, the modified drum may be placed, seated into the clay impoundment liner, and the water contained inside the modified drum may be removed using a peristaltic pumping system. The excess water will be returned to the impoundment through the discharge line of the system. When sufficient water is removed from the sampling location within the drum, a three-inch stainless steel AMS coring tool will be inserted into the sediment. The AMS tool will be seated into sediment until the tool meets resistance. At this time, it will be assumed that the tool has contacted the clay liner. The sampling team will continue the insertion until approximately 0.1 foot of clay is contained in the tool. The team will then remove the tool from the pond. (Note: The sampling team should use caution when removing the tool. The butterfly closure on the tool should provide a seal, but the team must ensure that all sample contents, including the 0.1 foot portion of the clay, is removed with the tool).

The sampled materials will be removed from the AMS tool using a stainless steel spatula and placed into a stainless steel holding tray. Field radiological measurements will be recorded at this time. The $0.1 \mathrm{ft}$. clay portion of the sample will be retained and homogenized with the remaining sample materials. Stainless steel tools and spatulas will be used to homogenize the materials. When initial homogenization is completed, the sampled materials will be transferred to the composite sample container. (Note: Simple field decontamination measures may be necessary to ensure contamination controls. If deemed necessary by the sampling team, or Radiation Protection personnel, sampling equipment may be rinsed during sampling activities using de-ionized water).

The methods described above will be repeated until all eighteen samples for each composite have been collected and placed into the composite sample container. Any excess impoundment water will be removed prior to homogenization of the composite sample. To facilitate the compositing and homogenizing process and provide better contamination control, moving the sample indoors is desired. Free standing water should be removed from the sample homogenization. The sample will be homogenized using stainless tools and steel spatulas. The homogenized sample will be packaged and prepared for laboratory delivery using the information contained in Table 2 of this SAP, QA requirements, and ORNL standard radiological protection procedures. All tools, equipment, and containers used for a sample shall be cleaned/ decontaminated and rinsed before reuse (see section 6.3.4).

\subsubsection{Field QC Samples}

When required by EPA standards, and when specified by the MAD Field Team Leader, Field QC samples will be collected during the sampling activities. Collect the QC samples in accordance with this SAP and applicable procedures. Sampling shall be consistent with EPA's Test Methods for Evaluating Solid Waste, SW-846, Third Edition. Preservation of equipment rinsate blanks (ERBs) and field duplicates shall adhere to the methods described in this SAP. Field Duplicates will be collected for a selected composite sample from each impoundment.

ERBs shall be collected on reusable sampling equipment. Collect one ERB prior to sampling activities on all reusable sampling equipment which is anticipated to be used in the sampling activities. At a specified time during sampling activities (preferably after relocating to the second impoundment), collect a second ERB on sampling equipment used during the sampling activities. A field duplicate sample will be collected from each impoundment for a selected composite sample. The MAD field team leader will specify the time and location of the collection of the composite duplicate sample.

A field blank will also be collected. The field blank sample will be collected from the source of the equipment decontamination water. The container and sample sizes for the Field QC samples are specified in Table 3. The field blank will be collected prior to sampling activities. 


\subsubsection{Sampling Equipment Decontamination}

Nondisposable sampling equipment shall be decontaminated and documented in accordance with Cleaning and Decontaminating Sample Containers and Sampling Devices, ESP-801.

\subsubsection{Sample Identification}

Samples will be identified using the following identification coding and qualifiers. Reference information for sample identifications can be found in the document Work Plan, Health and Safety Plan, and Quality Assurance Project Plan for K-1420 Waste Area Grouping, K/ER-34.

\subsubsection{Identification Numbering}

Project Code(s): $\quad$ SIOU39 $=$ SIOU 3539 Designation

SIOU40 $=$ SIOU 3540 Designation

Qualifiers: Sediment/Clay random composite sample designation $=\mathrm{COMP \#}$

Sediment/Clay systematic composite $=$ East or West

Field Duplicate Designation = DUP

Equipment Rinsate Blank Designation $=$ ERB

Field Blank Designation $=$ FB

Examples: Sediment/Clay Sample: SIOU39COMP1 = SIOU 3539 Composite Sample \#1

SIOU $40 \mathrm{West}=$ SIOU 3540 West side composite sample

Field QC Samples: $\quad$ SIOU40ERB2 = SIOU 3540 Equipment Rinsate Blank Sample \#2

SIOUFB1 = SIOU Project Field Blank Sample \#1

\subsubsection{Sample Labels}

Sample labeling will be conducted in accordance with this SAP and Quality Assurance Plan for the Oak Ridge National Laboratory Environmental Restoration Program, ORNL/ER-225/R1. Appropriate sample labels (e.g., Analytical, PCB, Radioactive) will be affixed to all sample containers prior to or at the time of sampling. To the extent practicable, sample bottles will be labeled prior to filling. Sample labels will be waterproof paper or plastic with gummed backs or waterproof tags, as appropriate. Labels will be completed with black indelible ink and will include, as a minimum, the following information:

- $\quad$ sample name;

- $\quad$ unique customer sample number (Note: Do not hyphenate customer sample numbers.);

- $\quad$ sampler's name;

- date and time of collection; and

- location of collection.

\subsubsection{Sample Chain of Custody}

The integrity of a sample from the time of sampling through receipt into the laboratory is assured by strict adherence to the Quality Assurance Plan for the Oak Ridge National Laboratory Environmental Restoration Program, ORNL/ER-225/RI. Copies of the completed chain-of-custody forms shall be provided to the MAD Field Team Leader upon completion of sampling activities, or after direct delivery to the laboratory. The following information, as a minimum, must be included on the chain-of-custody forms:

- unique sample number;

- $\quad$ signature of sample collector; 


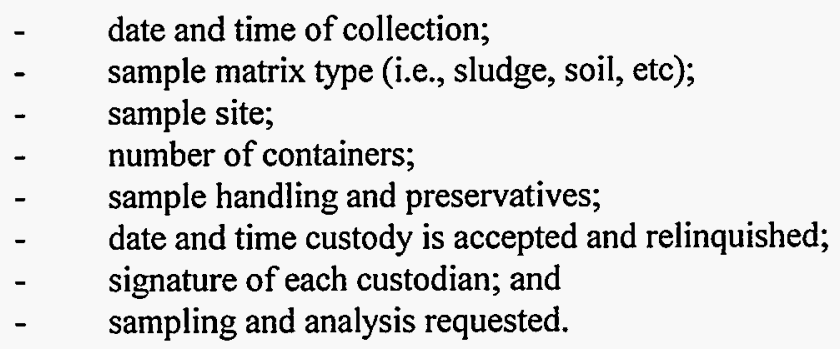

\subsubsection{Tamper Indicator Device}

A tamper indicator device (TID) assures that unauthorized additions to a container's contents can be visually detected. The TID shall be placed in a manner such that opening the container breaks the seal. A TID shall be placed on the following containers:

- $\quad$ containers that were sampled;

- $\quad$ analytical samples not delivered to the laboratory within 24 hours of sampling or not stored in a secure storage facility; and

- $\quad$ storage and transport packages such as ice coolers, etc.

\subsubsection{Sample Management}

The condition of samples (e.g., temperature, presence of TIDs, hazard labels, samples shipped on ice, etc.) shall be checked and documented by laboratory personnel upon arrival at the laboratory. Samples shall be stored at the laboratory at $4 \pm 2$ degrees Celsius.

\section{ANALYTICAL REQUIREMENTS}

The Analytical Coordinator will ensure that all analyses are performed in accordance with this SAP and all QC is performed in accordance with SW-846 and Analytical Master Specifications, dated September 1996, and this SAP. The primary regulatory methods for compliance are the applicable methods described in SW-846. Any deviations from specified methods or parameters must be approved and documented by the Project Manager or his/her designee prior to making changes. Analytical activities shall not be initiated prior to receipt of a signed copy of the SAP. The laboratory shall:

- $\quad$ archive all samples for 60 days;

- $\quad$ notify the MAD Field Team Leader immediately of any QC failures that would require resampling;

- $\quad$ report data on an as-received basis unless otherwise specified; and

- representatively obtain subsamples for analysis, rather than attempting to select the "cleanest" or "dirtiest" portion of the sample; and

- $\quad$ supply Level 4 package for analysis data.

\section{QUALITY ASSURANCE (QA) REQUIREMENTS}

At the direction of the Project Management, selected portions of a SAP prepared by Jacobs Engineering of Oak Ridge, TN (dated October 1997) were incorporated into this SAP. The selected portions include Attachment C of this plan (which deals with the statistical random start grids for sample collections) and the analyte requirement information in Table 2 and Table 3. Any exceptions or deviations to this plan require authorization from the Project Manager and MAD Field Team Leader. The stated sample collection procedure, Collection of Sediment Samples, ESP-304-1, Rev. 1, dated February 28, 1996, may be "red-lined" during activities to reflect actual sample collection methods used by the sampling team. The "red-line" instances will be documented in the project field logbook and must be approved by the MAD Field Team Leader.

Authorizations for deviations to procedures and this SAP can be made via telephone, verbal communications, or written instructions. When authorization is other than written correspondence, the Project Manager or MAD Field 
Team Leader shall document the date, requestor's name, and the deviation or exception. Deviations from analytical activities shall be approved by the Project Manager prior to implementation and documented by the Analytical Coordinator. Nonconforming items shall be administered by guidance in the Health Sciences Research Division Management Plan, MP-96-HSRD-001.

The QA Specialist or his/her designee may conduct a surveillance during sampling operations. Requirements such as chain-of-custody, sample labels, tamper-proof seals, field logbook entries, and the collection of QC samples will be reviewed for compliance to the procedures mentioned in this SAP. This surveillance report and any corrective action documentation will become part of the program's QA records. QA and other relevant project documentation will be maintained with the MAD Group for a minimum of three years.

The Analytical Coordinator will ensure that analytical data obtained complies with applicable SW-846 regulatory methods, Analytical Master Specifications, dated September 1996, and this SAP. The primary regulatory methods for compliance are the applicable methods described in SW-846.

In addition to the above quality controls measures, the project will follow the guidelines and requirements of the Quality Assurance Plan for the Oak Ridge National Laboratory Environmental Restoration Program, ORNL/ER225/R1.

\section{DATA DELIVERABLES}

The data deliverables for this project will be Level 4. The Analytical Coordinator will ensure that analytical data is reported to the appropriate personnel. All analytical results (Level 3) will be provided as they become available. Complete data packages (Level 4) will be provided within 30 days of the initial analytical results.

\section{DATA REVIEW AND VALIDATION}

The data package for this project will be validated according to Level 3 requirements as outlined in the Analytical Master Specification. Ten percent of the data packages will be validated according to Level 4 requirements of the Analytical Master Specification. If there are no major quality control concerns, no further validation will be required. If major quality control concerns are found, additional validation will be required. A validation report will be prepared and provided to the Project Manager.

\section{HEALTH AND SAFETY (H\&S)}

Health and Safety (H\&S) support will be obtained from site H\&S organizations. Preliminary reviews (e.g., Site Safety Reviews, Radiation Work Permits, etc.), requests for H\&S services, and establishment of communication with $\mathrm{H} \& \mathrm{~S}$ organizations are addressed in the project-specific addendum for the original Health and Safety Plan, WAG 1 Surface Impoundments Operable Unit (SIOU) Treatability Study, SSHASP No 001-226/0010 0396. The listing for the new personnel is contained in the H\&S addendum prepared for the project. An additional SSHO will be appointed for the project to act as an alternate and assist in any scheduling conflicts that may arise during the project activities. No additional hazards other than those addressed in the original plan are anticipated. 


\section{REFERENCES}

Cleaning and Decontaminating Sample Containers and Sampling Devices. ESP-801. Revision 0. Martin Marietta Energy Systems. August 27, 1988.

Analytical Master Specification, Sample Management Organization, draft dated September 1996.

Test Methods for Evaluating Solid Waste. SW-846, Third Edition. Environmental Protection Agency. November 1986.

Quality Assurance Plan for the Oak Ridge National Laboratory Environmental Restoration Program, ORNL/ER225/R1.

Collection of Sediment Samples, ESP-304-1, Rev. 1. February 28, 1996.

Health Sciences Research Division Management Plan, MP-96-HSRD-001, January 1996. 


\section{Pond 3539}

\begin{tabular}{|c|c|c|c|c|c|c|c|c|}
\hline & Compc & site \#1 & Compo & ite \#2 & Compos & jite \#3 & Compo & site \#4 \\
\hline & Easting & Northing & Easting & Northing & Easting & Northing & Easting & Northing \\
\hline 1 & 31302 & 21346 & 31342 & 21356 & 31340 & 21370 & 31336 & 21348 \\
\hline 2 & 31324 & 21348 & 31328 & 21366 & 31350 & 21340 & 31360 & 21360 \\
\hline 3 & 31330 & 21340 & 31342 & 21366 & 31352 & 21344 & 31334 & 21346 \\
\hline 4 & 31328 & 21368 & 31326 & 21348 & 31324 & 21362 & 31362 & 21350 \\
\hline 5 & 31354 & 21358 & 31308 & 21356 & 31352 & 21362 & 31310 & 21354 \\
\hline 6 & 31312 & 21346 & 31306 & 21362 & 31356 & 21348 & 31330 & 21348 \\
\hline 7 & 31306 & 21354 & 31346 & 21346 & 31338 & 21354 & 31350 & 21366 \\
\hline 8 & 31326 & 21350 & 31316 & 21346 & 31336 & 21360 & 31318 & 21348 \\
\hline 9 & 31330 & 21342 & 31350 & 21368 & 31360 & 21340 & 31356 & 21362 \\
\hline 10 & 31328 & 21370 & 31306 & 21352 & 31322 & 21356 & 31340 & 21352 \\
\hline 11 & 31362 & 21362 & 31304 & 21366 & 31312 & 21360 & 31360 & 21354 \\
\hline 12 & 31356 & 21358 & 31310 & 21368 & 31326 & 21362 & 31354 & 21368 \\
\hline 13 & 31306 & 21360 & 31328 & 21358 & 31352 & 21346 & 31322 & 21344 \\
\hline 14 & 31314 & 21352 & 31340 & 21354 & 31362 & 21354 & 31328 & 21356 \\
\hline 15 & 31346 & 21368 & 31320 & 21352 & 31308 & 21358 & 31302 & 21362 \\
\hline 16 & 31342 & 21360 & 31324 & 21340 & 31344 & 21350 & 31358 & 21368 \\
\hline 17 & 31302 & 21352 & 31358 & 21366 & 31356 & 21352 & 31350 & 21360 \\
\hline 18 & 31320 & 21346 & 31332 & 21360 & 31340 & 21346 & 31316 & 21368 \\
\hline 19 & 31322 & 21352 & 31346 & 21348 & 31332 & 21356 & 31336 & 21368 \\
\hline 20 & 31312 & 21368 & 31356 & 21348 & 31358 & 21352 & 31318 & 21350 \\
\hline 21 & 31336 & 21356 & 31314 & 21368 & 31328 & 21368 & 31332 & 21364 \\
\hline 22 & 31354 & 21348 & 31330 & 21366 & 31316 & 21364 & 31310 & 21366 \\
\hline 23 & 31362 & 21368 & 31356 & 21348 & 31308 & 21368 & 31348 & 21348 \\
\hline 24 & 31324 & 21340 & 31326 & 21344 & 31348 & 21364 & 31342 & 21360 \\
\hline 25 & 31326 & 21354 & 31332 & 21364 & 31314 & 21364 & 31348 & 21352 \\
\hline 26 & 31334 & 21342 & 31348 & 21358 & 31320 & 21356 & 31306 & 21352 \\
\hline 27 & 31356 & 21350 & 31316 & 21358 & 31344 & 21366 & 31310 & 21364 \\
\hline 28 & 31312 & 21370 & 31324 & 21368 & 31348 & 21366 & 31332 & 21356 \\
\hline 29 & 31332 & 21362 & 31310 & 21368 & 31316 & 21352 & 31338 & 21352 \\
\hline 30 & 31354 & 21368 & & & 31336 & 21366 & & \\
\hline
\end{tabular}




\section{Attachment A}

(continued)
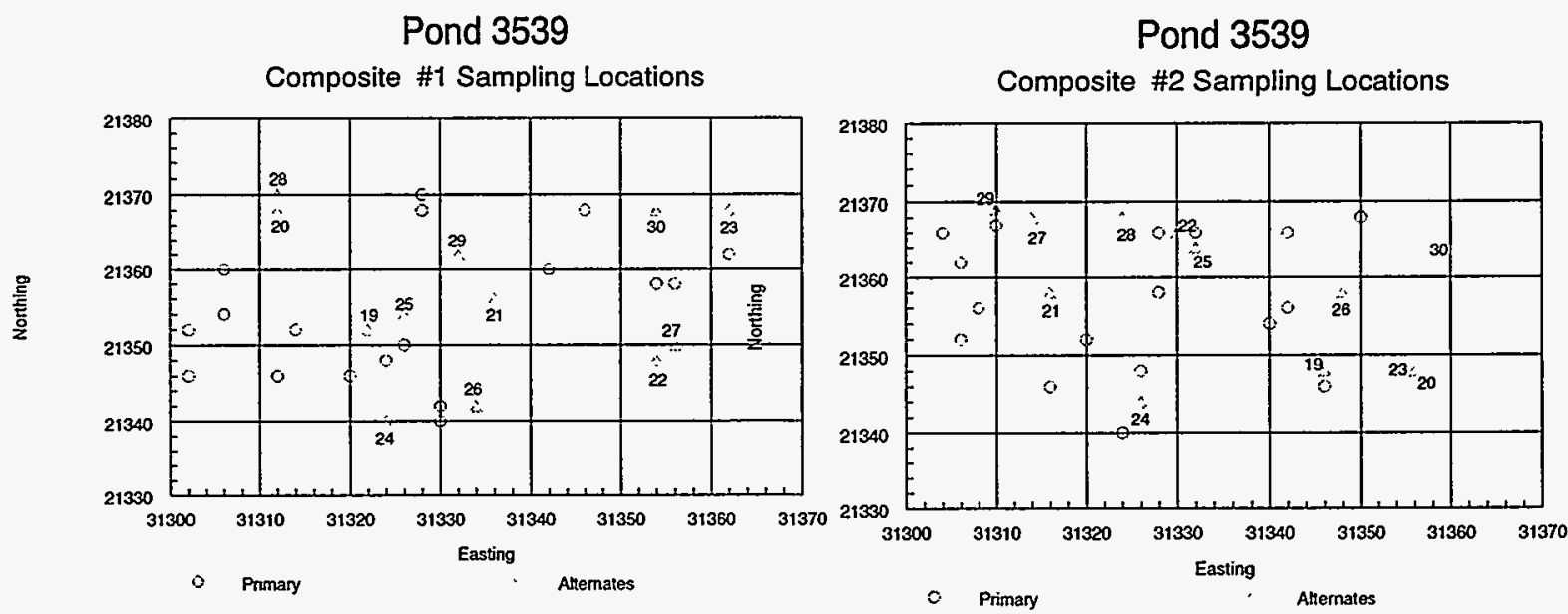

Pond 3539

Composite \#3 Sampling Locations

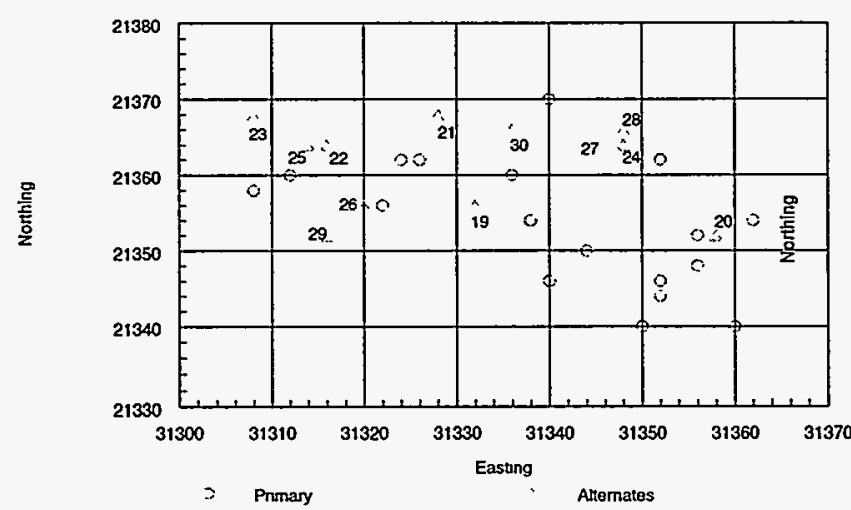

Pond 3539

Composite \#4 Sampling Locations

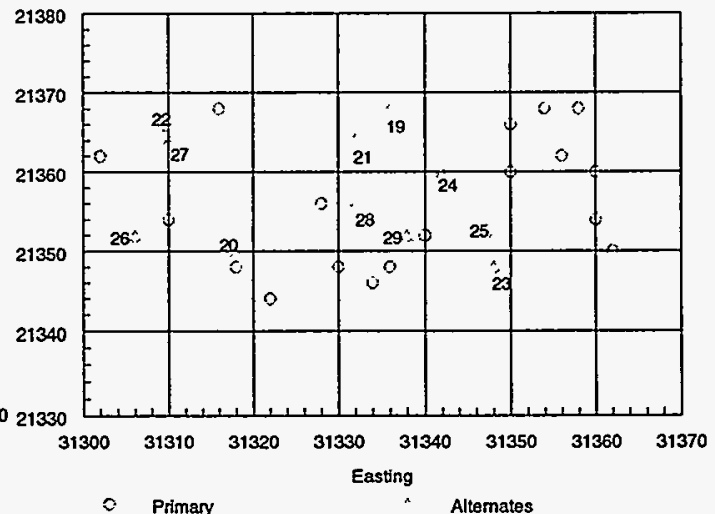




\section{Attachment B \\ Pond 3540}

\begin{tabular}{|c|c|c|c|c|c|c|c|c|}
\hline \multirow[b]{2}{*}{ Point \# } & \multicolumn{2}{|c|}{ Composite \#1 } & \multicolumn{2}{|c|}{ Composite \#2 } & \multicolumn{2}{|c|}{ Composite \#3 } & \multicolumn{2}{|c|}{ Composite \#4 } \\
\hline & Easting & Northing & Easting & Northing & Easting & Northing & Easting & Northing \\
\hline 1 & 31336 & 21288 & 31332 & 21290 & 31350 & 21280 & 31328 & 21300 \\
\hline 2 & 31326 & 21276 & 31314 & 21294 & 31342 & 21298 & 31360 & 21294 \\
\hline 3 & 31328 & 21276 & 31358 & 21286 & 31338 & 21278 & 31336 & 21280 \\
\hline 4 & 31322 & 21286 & 31312 & 21296 & 31330 & 21288 & 31314 & 21296 \\
\hline 5 & 31312 & 21286 & 31328 & 21284 & 31322 & 21278 & 31342 & 21300 \\
\hline 6 & 31328 & 21298 & 31326 & 21288 & 31350 & 21294 & 31334 & 21292 \\
\hline 7 & 31310 & 21290 & 31342 & 21286 & 31316 & 21296 & 31332 & 21292 \\
\hline 8 & 31340 & 21292 & 31358 & 21280 & 31324 & 21294 & 31328 & 21294 \\
\hline 9 & 31322 & 21280 & 31358 & 21300 & 31318 & 21282 & 31338 & 21292 \\
\hline 10 & 31322 & 21290 & 31336 & 21292 & 31322 & 21276 & 31322 & 21292 \\
\hline 11 & 31332 & 21278 & 31344 & 21280 & 31318 & 21278 & 31322 & 21302 \\
\hline 12 & .31306 & 21300 & 31320 & 21284 & 31352 & 21286 & 31332 & 21294 \\
\hline 13 & 31322 & 21296 & 31348 & 21296 & 31328 & 21288 & 31332 & 21288 \\
\hline 14 & 31358 & 21302 & 31338 & 21290 & 31338 & 21282 & 31346 & 21288 \\
\hline 15 & 31314 & 21284 & 31306 & 21294 & 31322 & 21298 & 31354 & 21292 \\
\hline 16 & 31310 & 21292 & 31360 & 21278 & 31358 & 21292 & 31320 & 21282 \\
\hline 17 & 31316 & 21290 & 31352 & 21290 & 31314 & 21280 & 31354 & 21298 \\
\hline 18 & 31358 & 21296 & 31324 & 21298 & 31360 & 21300 & 31322 & 21282 \\
\hline 19 & 31338 & 21290 & 31328 & 21280 & 31342 & 21278 & 31346 & 21302 \\
\hline 20 & 31348 & 21280 & 31346 & 21298 & 31316 & 21278 & 31348 & 21296 \\
\hline 21 & 31312 & 21284 & 31314 & 21278 & 31350 & 21282 & 31336 & 21302 \\
\hline 22 & 31314 & 21286 & 31344 & 21276 & 31320 & 21298 & 31344 & 21280 \\
\hline 23 & 31320 & 21300 & 31338 & 21296 & 31330 & 21284 & 31356 & 21292 \\
\hline 24 & 31348 & 21282 & 31330 & 21298 & 31306 & 21302 & 31308 & 21284 \\
\hline 25 & 31354 & 21294 & 31324 & 21300 & 31328 & 21280 & 31320 & 21280 \\
\hline 26 & 31334 & 21290 & 31348 & 21278 & 31310 & 21302 & 31328 & 21280 \\
\hline 27 & 31324 & 21282 & 31316 & 21302 & 31344 & 21296 & 31326 & 21294 \\
\hline 28 & 31350 & 21290 & 31332 & 21292 & 31360 & 21286 & 31348 & 21298 \\
\hline 29 & 31334 & 21294 & & & 31356 & 21296 & 31340 & 21302 \\
\hline 30 & 31350 & 21286 & & & 31306 & 21290 & & \\
\hline
\end{tabular}




\section{Attachment B}

(continued)

Pond 3540

Composite \#1 Sampling Locations

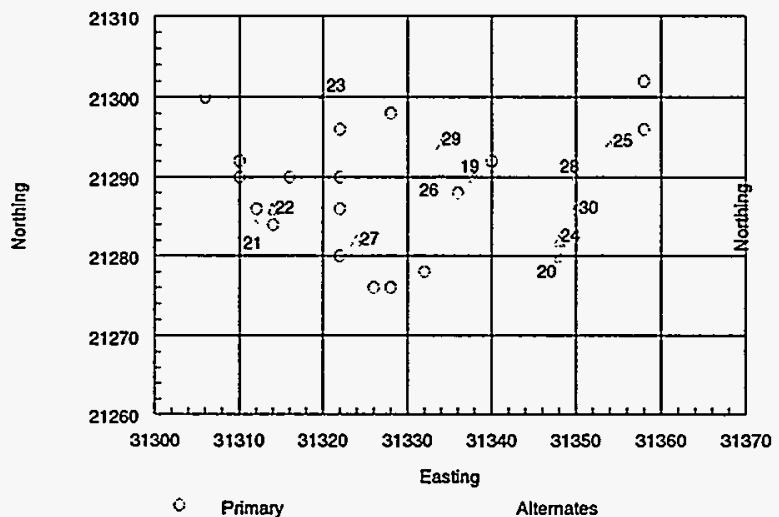

\section{Pond 3540}

Composite \#3 Sampling Locations

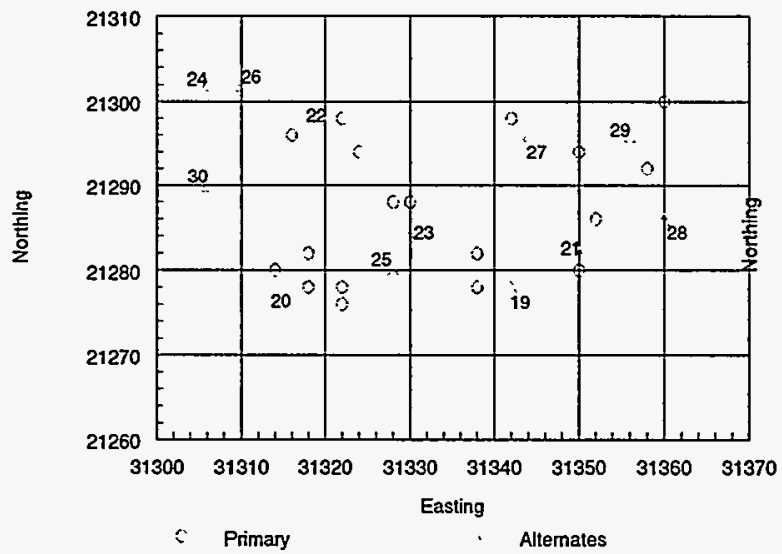

Pond 3540

Composite \#2 Sampling Locations

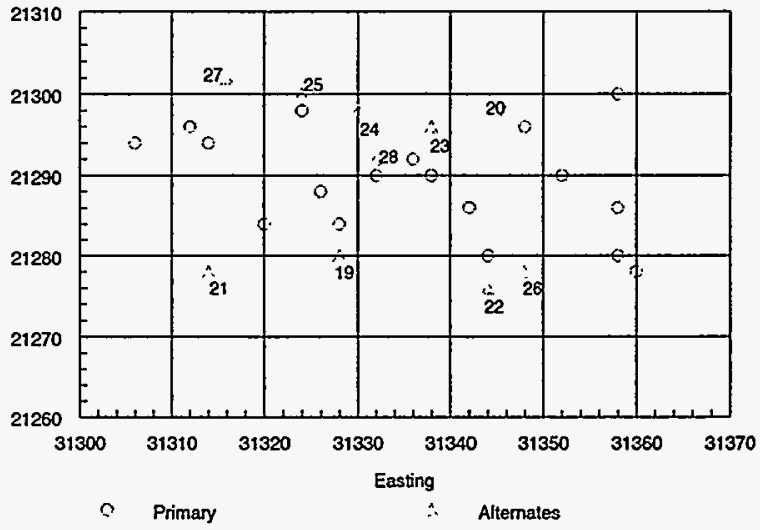

Pond 3540

Composite \#4 Sampling Locations

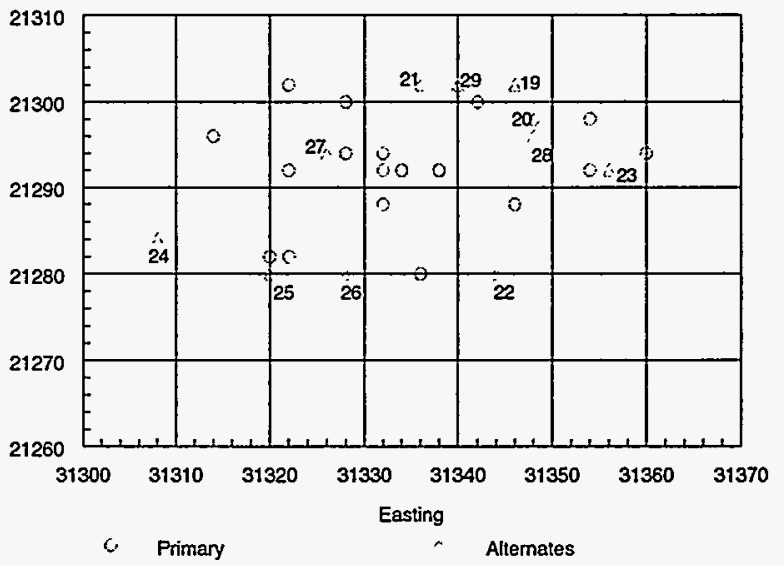


Attachment C.

Jacobs Engineering

Jacobs EM Team

CALCULATION PACKAGE COVER SHEET

Name of Assembler: G. John Hampshire

Date: October 15,1997

Task/Task Designator: 35 H83040, Surface Impoundments Operable Unit (SIOU)

Calculation Description: Calculation of the number of individual core samples to obtain in composite samples to be

taken from Impoundments 3539 and 3540.

References:

C.W. Francis and Sealand, O.M. September 1987. Concentrations of Radionuclides in ORNL Wastepond Sediments and Their Leaching Characteristics. Oak Ridge National Laboratory, Oak Ridge, Tennessee. ORNL/RAP/LTR-87/70.

DOE. November 1995. Remedial Investigation/Feasibility Study for Surface Impoundment Operable Unit, Waste Area Grouping 1, Oak Ridge National Laboratory, Oak Ridge, Tennessee. DOE/OR/02-1346\&D2.

U.S. EPA. September 1986. Test Methods for Evaluating Solid Waste: Physical/Chemical Methods, Chapter 9: Sampling Plan. EPA/SW/846 (Rev. 0).

Assumptions:

See attached sheets.

\begin{tabular}{|l|l|l|l|}
\hline \multicolumn{1}{|c|}{ Checked By } & Date & Approved By & Date \\
\hline & & & \\
\hline Keywords: & & & \\
SIOU ROD & & \\
\hline
\end{tabular}




\section{SIOU Sample Number Requirements for Impoundments 3539 and 3540}

Past investigations have only yielded one sample result for PCBs from each of these two impoundments. Furthermore, the methodology used to obtain these samples was a field modification of the sampling plan employing a scooping device being dragged along the impoundment bottom until sufficient volumes were collected, as well as reporting dry-weight-basis concentrations. The representativeness of this sample methodology to the expected method of waste generation from these impoundments is suspect, and it is therefore desired to obtain additional sample results to recharacterize these sediments. The methodology to be employed will obtain random, multiple-plug composite samples for each impoundment, to include 0.1 feet of subsurface clay liner (consistent with the stated expectations of the signed SIOU ROD). The following calculations attempt to estimate the number of individual plugs desired for each composite sample.

1. Previous sample results.

Although the representativeness of the previous sample results is suspect, they still represent the only measurement of PCB concentrations to date. Therefore, the information from these samples will be used to gain a rudimentary estimate of potential waste concentrations.

Previous sample results

\begin{tabular}{||l|l|l||}
\hline PCB Isomer & Impoundment 3539 & Impoundment 3540 \\
\hline Aroclor-1254 & $150 \mathrm{mg} / \mathrm{kg}$ (dry-weight) & $180 \mathrm{mg} / \mathrm{kg}$ (dry-weight) \\
\hline Aroclor-1260 & Not detected & $120 \mathrm{mg} / \mathrm{kg}$ (dry-weight) \\
\hline
\end{tabular}

It is notable that the sample concentrations for Aroclor-1260 are significantly different between the two impoundments. It is generally assumed that the two impoundments, which have had very similar operating histories, should have very similar contaminant concentrations. Therefore, either the assumption that the impoundments are similar is incorrect, or the sample results for Aroclor- 1260 are incorrect. In order to derive worst-case (i.e., maximum concentration) estimates, this calculation replaces the non-detect result with a value proportional to the ratio of Aroclor- 1260 to Aroclor1254 seen in Impoundment 3540.

$$
\text { Aroclor-1260 (Impoundment 3539) }=120 \mathrm{mg} / \mathrm{kg}\left(\frac{150 \mathrm{mg} / \mathrm{k}_{\mathrm{i}}}{180 \mathrm{mg} / \mathrm{k}_{\mathrm{i}}}\right.
$$

\section{Conversion to wet-weight-basis concentrations.}

Assuming the sediments are $20 \%$ solids and $80 \%$ water, and assuming the bulk density of the sediments is $1.2 \mathrm{~g} / \mathrm{cc}$ (wet-weight-basis)(per Francis \& Sealand 1987):

specific gravity of dry sediments $=(1.2 \mathrm{~g} / \mathrm{cc}-(80 \% \times 1.0$.

Converting dry-weight concentrations to wet-weight (i.e., as found) concentrations, assuming the concentration of PCBs in the interstitial water $=0 \mathrm{mg} / \mathrm{kg}$ :

$$
\text { wet-weight } \mathrm{mg} / \mathrm{kg}=\frac{(\mathrm{dry} \text {-weight } \mathrm{mg} / \mathrm{kg})(2.0 \mathrm{~g} / \mathrm{cc})(20 \%)+(}{1.2 \mathrm{~g} / \mathrm{cc}}
$$

Previous sample results re-expressed as wet-weight concentrations

\begin{tabular}{||l|l|l||}
\hline PCB Isomer & Impoundment 3539 & Impoundment 3540 \\
\hline Aroclor-1254 & $50 \mathrm{mg} / \mathrm{kg}$ (wet-weight) & $60 \mathrm{mg} / \mathrm{kg}$ (wet-weight) \\
\hline Aroclor-1260 & $33 \mathrm{mg} / \mathrm{kg}$ (wet-weight) & $40 \mathrm{mg} / \mathrm{kg}$ (wet-weight) \\
\hline
\end{tabular}

3. Estimation of the data variability. 
Since only one sample exists for each impoundment, there is no direct basis for estimating the variability of the concentrations in these impoundments. Therefore, the variability must be based on assumptions, and the effect of these assumptions needs to be examined in an uncertainty analysis. One overriding assumption for these impoundments is that, due to their relatively small size, the deposition of contaminants within each impoundment is somewhat uniform, such that normal statistics are applicable. It is also assumed that total PCB concentrations equal the sum of the individual detected isomer concentrations. It is uncertain what effect the additional clay material will have on the final sample concentration. It is possible that the clay has accumulated and concentrated PCBs, and will have higher concentrations than the overlying sediments. It is also possible that the clay will be devoid of PCB contaminants. For this calculation, it is assumed that the clay concentration equals that of the overlying sediment.

Total PCBs $=($ Aroclor $-1254(\mathrm{mg} / \mathrm{kg})+$ Aroclor $-1260(\mathrm{mg} / \mathrm{i}$

a. Assuming the standard deviation equals $1 / 4$ th of the mean:

\begin{tabular}{||l|l|l||}
\hline \hline PCB Isomer & Impoundment 3539 & Impoundment 3540 \\
\hline Aroclor-1254 & $50+/-12.5 \mathrm{mg} / \mathrm{kg}$ & $60+/-15 \mathrm{mg} / \mathrm{kg}$ \\
\hline Aroclor-1260 & $33+/-8.25 \mathrm{mg} / \mathrm{kg}$ & $40+/-10 \mathrm{mg} / \mathrm{kg}$ \\
\hline Total PCBs & $83+/-15 \mathrm{mg} / \mathrm{kg}$ & $100+/-18 \mathrm{mg} / \mathrm{kg}$ \\
\hline
\end{tabular}

b. Assuming the standard deviation equals half of the mean:

\begin{tabular}{||l|l|l||}
\hline PCB Isomer & Impoundment 3539 & Impoundment 3540 \\
\hline Aroclor-1254 & $50+/-25 \mathrm{mg} / \mathrm{kg}$ & $60+/-30 \mathrm{mg} / \mathrm{kg}$ \\
\hline Aroclor-1260 & $33+/-16.5 \mathrm{mg} / \mathrm{kg}$ & $40+/-20 \mathrm{mg} / \mathrm{kg}$ \\
\hline Total PCBs & $83+/-30 \mathrm{mg} / \mathrm{kg}$ & $100+/-36 \mathrm{mg} / \mathrm{kg}$ \\
\hline
\end{tabular}

c. Assuming the standard deviation equals the mean:

\begin{tabular}{||l|l|l||}
\hline PCB Isomer & Impoundment 3539 & Impoundment 3540 \\
\hline Aroclor-1254 & $50+/-50 \mathrm{mg} / \mathrm{kg}$ & $60+/-60 \mathrm{mg} / \mathrm{kg}$ \\
\hline Aroclor-1260 & $33+/-33 \mathrm{mg} / \mathrm{kg}$ & $40+/-40 \mathrm{mg} / \mathrm{kg}$ \\
\hline Total PCBs & $83+/-60 \mathrm{mg} / \mathrm{kg}$ & $100+/-72 \mathrm{mg} / \mathrm{kg}$ \\
\hline
\end{tabular}

4. Calculation of the numbers of cores to combine into a composite sample.

The numbers of individual core samples that make up a composite sample are theoretically linked to the confidence that the composite sample result equals the population mean. In other words, the more cores taken randomly across the impoundment, the closer the result should be to the actual mean. In order to express this relationship, the number of cores to theoretically obtain various relative fractional differences between the $\mathrm{UCL}_{95}$ and the mean is used.

$$
U C L_{95}=\bar{x}+t_{(0.05, n-1, \text { one-tailed })} \frac{\sigma}{\sqrt{n}}
$$

$$
\frac{((1+\text { relative fractional difference }) \bar{x}-\bar{x})}{\sigma}=\frac{t_{(0.05, n-1, \text { one-tailed })}}{\sqrt{n}}
$$




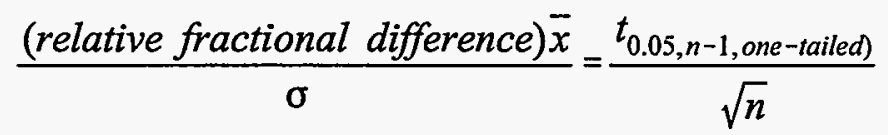

The following attached sheet details the evaluation of the required number of cores to give a $95 \%$ confidence that the $\mathrm{UCL}_{95}$ of each data set is within a given relative fractional difference of its mean. These results can also be interpreted as a concentration range over which multiple composite samples would fall if they were drawn from a population represented by these averages and standard deviations. Such an interpretation would take the form of an expectation for $90 \%$ of the composite results to fall within a range of plus or minus the relative fractional difference expected for a given number of cores.

\section{Selection of the number of cores to obtain for each composite sample.}

The number of cores obtained for each sample must balance practical considerations such as time to obtain samples and limitations on the amounts of material that can be effectively composited, with technical considerations, such as the expected representativeness of the composite sample to the actual population mean. The uncertainties associated with the estimates of the population variability also need to be considered.

A review of the results of these analyses indicates 18 cores per composite sample would be desirable. This number of samples would yield less than a 10\% (approximately $7 \%$ ) relative fractional difference between the composite result and its expected $\mathrm{UCL}_{95}$ if the population standard deviation was $1 / 4$ th of the population mean, a $15 \%$ relative fractional difference for a population where the standard deviation is half the population mean, and a $30 \%$ relative fractional difference for a population where the standard deviation is equal to the population mean. Fewer cores than this may result in unacceptable relative fractional differences if the population in fact has a large standard deviation, while significantly more cores than this may be difficult to justify from a cost-benefit and practicality perspective. The maximum suggested number of cores is 38 for similar reasons.

6. Numbers of composite samples and final data usage.

The following basic equation in EPA's SW-846 guidance was used to determine the maximum concentrations that would be considered as less than the regulatory limit of $50 \mathrm{ppm}$ total PCBs for various hypothesized relative fractional differences and numbers of sample taken.

$$
\text { number of samples }=\frac{t^{2}{ }_{(0.20, n-1, \text { two-tailed })} s^{2}}{\text { (regulatory threshold }- \text { population } m \iota}
$$

Also attached is an analysis of the effect of various numbers of composite samples that could be obtained from each impoundment on the mean concentration of those composite samples that could be construed as meeting the release limit (assuming that no individual sample exceeded the release limit). As can be seen, a total of four composite samples yields an acceptable degree of conservatism (i.e., an average concentration that is reasonably close to $50 \mathrm{ppm}$ ) for the least number of samples. It also gives a degree of assurance that acceptable release limits could be derived should one of the composite samples be rejected for failing to meet quality assurance/quality control requirements. Beyond four samples, the next significant gain that would be achieved would not be realized until 10 samples were taken which, given the small size of these impoundments, is deemed to be impractical. In total, four composite samples with 18 individual cores per sample would yield 72 individual cores per impoundment, a significant coverage for these 60 -foot by 90 -foot structures.

Furthermore, since uncertainties are introduced by the potential for improper compositing, it is suggested that field duplicate samples be obtained for each of the composites. If the relative fractional difference between the composites exceeds $20 \%$, it is suggested that the data from that particular composite sample be considered suspect (i.e., potentially unreliable). Otherwise, the average of the two results should be used as the best estimate of the actual concentration in each composite sample set.

Once validated results are obtained, it is suggested that the average results of each composite sample be compared to the regulatory threshold of $50 \mathrm{ppm}$ total PCBs. If any one of these results exceeds $50 \mathrm{ppm}$ total PCBs, the impoundment would be concluded to contain TSCA-regulated material. If all results meet this threshold criterion, a secondary criterion 
for the UCL $\mathrm{UCF}_{90}$ of the results to be less than $50 \mathrm{ppm}$ (consistent with guidance in EPA SW-846) would have to be met before the impoundments would be considered as not regulated under TSCA. 


\begin{tabular}{|c|c|c|c|c|c|c|c|c|c|c|c|c|c|}
\hline \multicolumn{6}{|c|}{ Assuming the standard deviation $=1 / 4$ the mean: } & \multicolumn{8}{|c|}{$t_{0,05, n-1, \text { onc-1ailod }} / \mathrm{sqrt}(\mathrm{n})$} \\
\hline \multicolumn{3}{|c|}{ Impoundment 3539} & \multicolumn{3}{|c|}{ Impoundment 3540} & & & 51 & 0.234674 & 101 & \begin{tabular}{|l|}
0.1652 \\
\end{tabular} & 151 & 0.134688 \\
\hline average & 83 & & average & 100 & & 2 & 4.464494 & 52 & 0.23232 & 102 & \begin{tabular}{|l|}
0.164372 \\
\end{tabular} & $\sim$ & \\
\hline \multirow[t]{2}{*}{ std dev } & 15 & & std dev & 18 & & 3 & 1.685855 & 53 & 0.230036 & 103 & 0.163558 & 560 & 0.069623 \\
\hline & & & & & & 4 & 1.176682 & 54 & 0.227818 & 104 & 0.162755 & 561 & 0.069561 \\
\hline $\begin{array}{l}\text { relative } \\
\text { difference }\end{array}$ & criterion & \begin{tabular}{|l|} 
number of \\
samples
\end{tabular} & \begin{tabular}{|l|} 
relative \\
difference
\end{tabular} & criterion & \begin{tabular}{|l|} 
number of \\
samples
\end{tabular} & 5 & 0.953391 & 55 & 0.225664 & 105 & 0.161964 & 562 & 0.069499 \\
\hline $30 \%$ & 1.66 & 4 & $30 \%$ & 1.6666667 & \begin{tabular}{|r|}
4 \\
\end{tabular} & 6 & 0.82264 & 56 & 0.223569 & 106 & 0.161184 & 563 & 0.069437 \\
\hline $25 \%$ & 1.383333 & 4 & $25 \%$ & 1.3888889 & 4 & 7 & 0.734453 & 57 & 0.221531 & 107 & 0.160416 & \begin{tabular}{|l|}
564 \\
\end{tabular} & 0.069375 \\
\hline $20 \%$ & 1.106667 & 5 & $20 \%$ & 1.1111111 & 5 & 8 & 0.669834 & 58 & 0.219548 & 108 & \begin{tabular}{|l|}
0.159658 \\
\end{tabular} & 565 & 0.069313 \\
\hline $15 \%$ & 0.83 & 6 & $15 \%$ & 0.8333333 & 6 & 9 & 0.619849 & 59 & 0.217618 & 109 & \begin{tabular}{|l|}
0.158912 \\
\end{tabular} & 566 & 0.069252 \\
\hline $10 \%$ & 0.553333 & 11 & $10 \%$ & 0.5555556 & 111 & 10 & 0.579681 & 60 & 0.215737 & 110 & 0.158175 & 567 & 0.069191 \\
\hline \multirow[t]{2}{*}{$5 \%$} & 0.276667 & 38 & $5 \%$ & 0.2777778 & 37 & 11 & 0.546478 & 61 & 0.213905 & 111 & 0.157448 & 568 & 0.069129 \\
\hline & & & & & & 12 & 0.518427 & 62 & 0.212118 & 112 & 0.156732 & 569 & 0.069068 \\
\hline \multicolumn{6}{|c|}{ Assuming the standard deviation $=1 / 2$ the mean: } & 13 & 0.494317 & 63 & 0.210376 & 113 & 0.156025 & 570 & 0.069008 \\
\hline \multicolumn{3}{|c|}{ Impoundment 3539} & \multicolumn{3}{|c|}{ Impoundment 3540} & 14 & 0.473301 & 64 & 0.208675 & 114 & 0.155328 & & \\
\hline average & 83 & & average & 100 & & 15 & 0.454768 & 65 & 0.207016 & 115 & 0.15464 & & \\
\hline \multirow[t]{2}{*}{ std dev } & 30 & & std dev & 36 & & 16 & 0.438263 & 66 & 0.205395 & 116 & 0.153961 & & \\
\hline & & & & & & 17 & 0.423439 & 67 & 0.203812 & 117 & 0.153291 & & \\
\hline $\begin{array}{l}\text { relative } \\
\text { difference }\end{array}$ & criterion & \begin{tabular}{|l|} 
number of \\
samples
\end{tabular} & \begin{tabular}{|l|} 
relative \\
difference
\end{tabular} & criterion & \begin{tabular}{|l|} 
number of \\
samples
\end{tabular} & 18 & 0.410029 & 68 & 0.202264 & 118 & 0.15263 & & \\
\hline $30 \%$ & \begin{tabular}{|l|}
0.83 \\
\end{tabular} & 6 & $30 \%$ & 0.8333333 & 6 & 19 & 0.397821 & 69 & 0.200752 & 119 & 0.151977 & & \\
\hline $25 \%$ & \begin{tabular}{|l|}
0.691667 \\
\end{tabular} & 8 & $25 \%$ & 0.6944444 & 8 & 20 & 0.386646 & 70 & 0.199273 & 120 & 0.151332 & & \\
\hline $20 \%$ & 0.553333 & 11 & $20 \%$ & 0.5555556 & 11 & 21 & 0.376364 & 71 & 0.197826 & 121 & 0.150695 & & \\
\hline $15 \%$ & 0.415 & 18 & $15 \%$ & 0.4166667 & 18 & 22 & 0.366864 & 72 & 0.196411 & 122 & \begin{tabular}{|l|}
0.150067 \\
\end{tabular} & & \\
\hline $10 \%$ & 0.276667 & 38 & $10 \%$ & 0.2777778 & 37 & 23 & 0.358049 & 73 & 0.195025 & 123 & 0.149446 & & \\
\hline \multirow[t]{2}{*}{$5 \%$} & 0.138333 & 144 & $5 \%$ & 0.1388889 & 143 & 24 & 0.349842 & 74 & 0.193668 & 124 & 0.148833 & & \\
\hline & & & & & & 25 & 0.342176 & 75 & 0.192339 & 125 & 0.148228 & & \\
\hline \multicolumn{6}{|c|}{ Assuming the standard deviation $=$ the mean: } & 26 & 0.334994 & 76 & 0.191037 & 126 & 0.147629 & & \\
\hline \multicolumn{3}{|c|}{ Impoundment 3539} & \multicolumn{3}{|c|}{ Impoundment 3540} & 27 & 0.328246 & 77 & 0.189762 & 127 & 0.147038 & & \\
\hline average & 83 & & average & 100 & & 28 & 0.321891 & 78 & 0.188511 & 128 & 0.146454 & & \\
\hline \multirow[t]{2}{*}{ std dev } & 60 & & std dev & 72 & & 29 & 0.315892 & 79 & 0.187285 & 129 & 0.145877 & & \\
\hline & & & & & & 30 & 0.310217 & 80 & 0.186082 & 130 & 0.145307 & & \\
\hline $\begin{array}{l}\text { relative } \\
\text { difference }\end{array}$ & criterion & $\begin{array}{l}\text { number of } \\
\text { samples }\end{array}$ & $\begin{array}{l}\text { relative } \\
\text { difference }\end{array}$ & criterion & \begin{tabular}{|l|} 
number of \\
samples
\end{tabular} & 31 & 0.304837 & 81 & 0.184903 & 131 & 0.144743 & & \\
\hline $30 \%$ & 0.415 & 18 & $30 \%$ & 0.4166667 & 18 & 32 & 0.299728 & 82 & 0.183745 & 132 & 0.144186 & & \\
\hline $25 \%$ & 0.345833 & 25 & $25 \%$ & 0.3472222 & 25 & 33 & 0.294868 & 83 & 0.182609 & 133 & 0.143635 & & \\
\hline $20 \%$ & 0.276667 & 38 & $20 \%$ & 0.2777778 & 37 & 34 & 0.290237 & 84 & 0.181494 & 134 & 0.14309 & & \\
\hline $15 \%$ & \begin{tabular}{|l|}
0.2075 \\
\end{tabular} & 65 & $15 \%$ & 0.2083333 & 65 & 35 & 0.285818 & 85 & 0.180399 & 135 & 0.142552 & & \\
\hline $10 \%$ & 0.138333 & 144 & $10 \%$ & 0.1388889 & 143 & 36 & 0.281595 & 86 & 0.179324 & 136 & 0.14202 & & \\
\hline \multirow[t]{14}{*}{$5 \%$} & 0.069167 & 568 & $5 \%$ & 0.0694444 & 563 & 37 & 0.277554 & 87 & 0.178267 & 137 & 0.141493 & & \\
\hline & & & & & & 38 & 0.273683 & 88 & 0.177229 & 138 & 0.140973 & & \\
\hline & & & & & & 39 & 0.269969 & 89 & 0.176209 & 139 & 0.140458 & & \\
\hline & & & & & & 40 & 0.266402 & 90 & 0.175207 & 140 & 0.139948 & & \\
\hline & & & & & & 41 & 0.262974 & 91 & 0.174221 & 141 & 0.139444 & & \\
\hline & & & & & & 42 & 0.259674 & 92 & 0.173252 & 142 & 0.138946 & & \\
\hline & & & & & & 43 & 0.256495 & 93 & 0.172298 & 143 & 0.138453 & & \\
\hline & & & & & & 44 & 0.253431 & 94 & 0.171361 & 144 & 0.137965 & & \\
\hline & & & & & & 45 & 0.250474 & 95 & 0.170438 & 145 & 0.137482 & & \\
\hline & & & & & & 46 & 0.247618 & 96 & 0.16953 & 146 & 0.137004 & & \\
\hline & & & & & & 47 & 0.244858 & 97 & 0.168637 & 147 & 0.136532 & & \\
\hline & & & & & & 48 & 0.242188 & 98 & 0.167758 & 148 & 0.136064 & & \\
\hline & & & & & & 49 & 0.239603 & 99 & 0.166892 & 149 & 0.1356 & & \\
\hline & & & & & & 50 & 0.2371 & 100 & 0.166039 & 150 & 0.135142 & & \\
\hline
\end{tabular}




\section{SIOU - Number of Composites}

\begin{tabular}{|c|c|c|c|c|c|c|c|}
\hline \multicolumn{2}{|c|}{ Numbers of composite samples } & \multicolumn{2}{|c|}{ assuming $s=0.3($ mean $) / 1.645$} & \multicolumn{2}{|c|}{ assuming $\mathrm{s}=0.15$ (mean) $/ 1.645$} & \multicolumn{2}{|c|}{ assuming $\mathrm{s}=0.07$ (mean) $/ 1.645$} \\
\hline$n$ & $n / t(.2, n-1)^{n}$ & $\begin{array}{l}\text { mean } \\
\text { concentration }\end{array}$ & $\begin{array}{l}\mathrm{s}^{\wedge} 2 / \text { delta }^{\wedge} 2 \\
\text { delta }=50-\mathrm{x}\end{array}$ & $\begin{array}{l}\text { mean } \\
\text { concentration }\end{array}$ & $\begin{array}{l}\mathbf{s}^{\wedge} 2 / \text { delta }^{\wedge} 2 \\
\text { delta }=50-\mathrm{x}\end{array}$ & $\begin{array}{l}\text { mean } \\
\text { concentration }\end{array}$ & $\begin{array}{l}\mathrm{s}^{\wedge} 2 / \text { delta }^{\wedge} 2 \\
\text { delta }=50-\mathrm{x}\end{array}$ \\
\hline 2 & 0.211145 & 3 & 0.18110978 & 35 & 0.04527744 & 3 & 0.00986042 \\
\hline 3 & 0.843749 & 3 & 0.21995673 & 36 & 0.05498918 & 3 & 0.01197542 \\
\hline 4 & 1.491308 & 3 & 0.26946668 & 37 & 0.06736667 & 3 & 0.01467096 \\
\hline 5 & 2.127008 & 3 & 0.33357464 & 38 & 0.08339366 & 3 & 0.01816129 \\
\hline$\overline{6}$ & 2.754523 & 3 & 0.41815007 & $\overline{39}$ & 0.10453752 & 3 & 0.02276595 \\
\hline 7 & 3.37692 & 4 & 0.53224098 & 40 & 0.13306024 & 4 & 0.02897756 \\
\hline 8 & 3.995986 & 4 & 0.69035269 & 41 & 0.17258817 & 4 & 0.03758587 \\
\hline 9 & 4.612797 & 4. & 0.91686825 & 42 & 0.22921706 & 4 & 0.04991838 \\
\hline 10 & 5.228024 & 4 & 1.2552469 & 43 & 0.31381173 & 4 & 0.06834122 \\
\hline 11 & 5.842085 & 4 & 1.78892107 & 44 & 0.44723027 & 4 & 0.09739681 \\
\hline 12 & 6.455284 & 4 & 2.69446996 & 45 & 0.67361749 & 4 & 0.14669892 \\
\hline 13 & 7.067801 & 4 & 4.39930434 & 46 & 1.09982609 & 4 & 0.23951768 \\
\hline 14 & 7.679797 & 47 & 8.16472446 & 47 & 2.04118112 & 4 & 0.44452389 \\
\hline 15 & 8.291374 & 48 & 19.1606752 & 48 & 4.79016881 & 4 & 1.04319232 \\
\hline 16 & 8.902624 & 4 & 79.8694119 & 49 & 19.967353 & 4 & 4.34844576 \\
\hline 17 & 9.513579 & & & & & & \\
\hline 18 & 10.1243 & & & & & & \\
\hline & & & & & & & \\
\hline
\end{tabular}


SIOU - Number of Composites

\begin{tabular}{|c|c|c|c|c|c|c|c|}
\hline \multicolumn{2}{|c|}{ Numbers of composite samples } & \multicolumn{2}{|c|}{ assuming $\mathrm{s}=0.3(\mathrm{mean}) / 1.645$} & \multicolumn{2}{|c|}{ assuming $s=0.15$ (mean) $/ 1.645$} & \multicolumn{2}{|c|}{ assuming $\mathrm{s}=0.07$ (mean) $/ 1.645$} \\
\hline 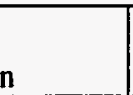 & $\mathrm{n} / \mathrm{t}(.2, \mathrm{n}-1)^{\wedge}$ & $\begin{array}{l}\text { mean } \\
\text { concentration }\end{array}$ & $\begin{array}{l}\mathrm{s}^{\wedge} 2 / \text { delta } 2, \\
\text { delta }=50-\mathrm{x}\end{array}$ & $\begin{array}{l}\text { mean } \\
\text { concentration }\end{array}$ & $\begin{array}{l}\mathrm{s}^{\wedge} 2 / \text { delta }^{\wedge} 2 \\
\text { delta }=50-\mathrm{x}\end{array}$ & $\begin{array}{l}\text { mean } \\
\text { concentration }\end{array}$ & $\begin{array}{l}\mathrm{s}^{\wedge} 2 / \text { delta } 2 \\
\text { delta }=50-\mathrm{x}\end{array}$ \\
\hline 2 & 0.211145 & 3 & 0.18110978 & 3 & 0.04527744 & & \begin{tabular}{|l|l|}
35 & 0.00986042 \\
\end{tabular} \\
\hline 3 & $\mid 0.843749$ & 3 & 0.21995673 & $\overline{3}$ & 0.05498918 & & 0.01197542 \\
\hline 4 & 1.491308 & 3 & 0.26946668 & 3 & 0.06736667 & & 0.01467096 \\
\hline 5 & 2.127008 & 38 & 0.33357464 & 3 & 0.08339366 & & 0.01816129 \\
\hline 6 & 2.754523 & 3 & 0.41815007 & $\overline{3}$ & 0.10453752 & & 0.02276595 \\
\hline 7 & 3.37692 & $\overline{4}$ & 0.53224098 & $\overline{4}$ & 0.13306024 & & 0.02897756 \\
\hline 8 & 3.995986 & 4 & 0.69035269 & 4 & 0.17258817 & & 0.03758587 \\
\hline 9 & \begin{tabular}{|l|}
4.612797 \\
\end{tabular} & 4. & 0.91686825 & $\overline{4}$ & 0.22921706 & & 0.04991838 \\
\hline 10 & 5.228024 & 4. & 1.2552469 & 4 & 0.31381173 & & 0.06834122 \\
\hline 11 & 5.842085 & 4 & 1.78892107 & 4 & 0.44723027 & & 0.09739681 \\
\hline 12 & $\mid 6.455284$ & 4 & 2.69446996 & $\overline{4}$ & 0.67361749 & & 0.14669892 \\
\hline 13 & 7.067801 & 4 & 4.39930434 & 4 & 1.09982609 & & 0.23951768 \\
\hline 14 & \begin{tabular}{|l|}
7.679797 \\
\end{tabular} & 4 & 8.16472446 & 4 & 2.04118112 & & 0.44452389 \\
\hline 15 & 8.291374 & 4 & 19.1606752 & 4 & 4.79016881 & & 1.04319232 \\
\hline 16 & 8.902624 & 4 & 79.8694119 & 4 & 19.967353 & & 4.34844576 \\
\hline 17 & 9.513579 & & & & & & \\
\hline 18 & 10.1243 & & & & & & \\
\hline & & & & & & & \\
\hline
\end{tabular}




\section{APPENDIX B}

\section{HEALTH AND SAFETY ADDENDUM}

Sediment Sampling, Impoundments SIOU 3539 and 3540 
HEALTH and SAFETY ADDENDUM

Date: November 12, 1997

Project: Sediment Sampling, Impoundments SIOU 3539 and 3540

Initiator of Form: D.E. Rice / D.A. Rose Phone : 576-8565/574-5837

Task or Hazard: ORNL/MAD personnel will collect and sample sediment materials from each of the impoundments. The samples will be analyzed for selected chemical and radiological constituents. ORNL / MAD personnel will perform these activities under the guidelines and requirements of the original Health \& Safety Plan (No. 001-226/0010 0396, completed and signed March 1996). The new task of sampling these impoundments will be performed using the guidelines and requirements of this addendum. The new task will require the use of a boat and other work over water will still be required. These hazards are covered under the original plan. No additional hazards other than those covered under the original H\&S plan are anticipated. Appropriate sampling tools will be devised and fabricated for the task. Samples will be contained, packaged, and analyzed following the guidelines of the project Sampling and Analysis Plan(SAP). Perimeter support services will be provided by P\&E and Health Physics personnel. A new "Roles and Responsibilities" list will be prepared due to changes in support personnel. The new list can be found in the project SAP, and will be available to on-site workers at all times.

\section{Project / Facility Managers Information}

\begin{tabular}{|ll|}
\hline Name: R.A. Dean & Phone / Pager Contact: 574-7951 / 873-4911 \\
\hline Name: Hollis Wooten & Phone / Pager Contact: 241-4872 / 873-5038 \\
\hline Name: R.H. Coe III & Phone / Pager Contact: 241-5026 / 873-4937 \\
\hline
\end{tabular}

\section{Additional Hazards:}

Physical Hazards
(x) Cold Stress
() Compressed Gas/Cylinders () Confined Space
() Enclosed Space
(x) Ergonomics
() Explosive/Flammable
(x) Heat Stress
() High Pressure
( ) Manual Lift
() Noise
() Oxygen Deficient
() Oxygen Enriched

(x) Tripping/ Falling

(x) Work On/Over Water (See administrative controls)

Safety and Construction Hazards
() Demolition
() Drum Handling
(x) Electrical (See additional comments)
(x) Elevated Work (See additional comments)
() Energized Sources (Lockout/ Tagout)
() Excavation/ Penetration
(x) Hoisting/ Rigging (See additional comments)
() Overhead Hazards
() Trenching/ Shoring
( ) Underground Hazards
() Welding/ Burning/ Cutting 
Chemical Hazards
() Asbestos
(x) Carcinogen
() Corrosive
() Inorganics
() Lead
() Manmade Mineral Fibers
(x) Mercury
(x) Metals
() Mutagen
() OSHA Specific
(x) PCBs
( ) Reproductive Toxicant
( ) Volatile Organic
() Other

Ionizing Radiological Hazards

(x) External Exposure

( ) Internal Exposure

(x) Contamination Hazard (Type: Beta-Gamma)

Note Possible Routes: () Ingestion, () Inhalation, () Absorption

Non-Ionizing Radiological Hazards
() High Voltage
() Laser
() Microwave
() $\mathrm{RF}$
() UV

Biological / Vector Hazards

() Bacterial

() Medical Wastes

() Parasites

(x) Plants (Allergens)

(x)

Wildlife (snakes, ticks, insects)

\section{Controls:}

\section{Engineering Controls:}

- Support personnel involved with engineering aspects of the tasks will be responsible for controls.

- A boat and walkboards with handrail support will be utilized during sample collections.

- Hoisting and rigging of the walkboards and the boat will be performed by the ORNL P\&E rigging crews. Tools will be designed and utilized to reduce radiological exposures to sampling personnel.

- Ropes should be placed across walkbeam access to prevent unauthorized entries and provide fall protection.

- Aluminum walkboards will be utilized if available and practical.

\section{Administrative Controls:}

- RCT will be responsible for controls such as RWP requirements, etc.

- ORNL / MAD will be responsible for daily activity controls such as access, sample collection activities, etc.

- Facility representative will be responsible for obtaining the appropriate work permits as required.

- Quality control measures will be addressed in the Sampling and Analysis Plan and will be the responsibility of the MAD sampling team.

- D.E. Rice will replace P. Abston as SSHO (original plan); D.A. Rose will be designated as the alternate

- All personnel will review and sign off on original H\&S plan. The signature list will be maintained in the project logbook.

- Pre-task and daily H\&S briefings will be performed by the SSHO or the SSHO alternate.

- For the work performed on water, the handrails and walkboards will be utilized as fall protection. Spotters will be on-site at all times during work on the water. A safety line will be available from the walkboard and zone boundaries.

\section{Permits Required}
(x) Radiation Work Permit
( ) Lockout / Tagout
( ) PACSE
( ) Excavation/ Penetration
( ) Hoisting/ Rigging

Are Changes Required in Existing Permits ? ( ) Yes

Are Design / Specification Needed ? ( ) Yes (x) No

(x) No

Other (Specify): A new RWP will be issued prior to the start of work.

Personal Protective Equipment: (Suggestions for minimum site access only, work to be performed under RWP PPE requirements) 

() Level "A"
() Level "B"
(x) Level "C"
(x) Level " $D$ "
() Modified

Respiratory Protection
() SCBA
() Full-face
() Half-face
() PAPR
() Supplied Air
() Other

Cartridge Type:

Protective Clothing

(x) Apron (Rubber if applicable) ( ) Impermeable Suit
() Saranex
(x) Tyvek
() Encapsulating Suit
( ) Splash Suit
() Welded Saranex
() Lab Coat

(x) Company Clothing (Khakis) () Other

() Other

Head/Eye/Ear
() Ear Plugs
() Ear Muffs
() Splash Shield
() Monogoggles
(x) Safety Glasses
() Welding Goggles
() Face Shield
() Goggles
( ) Hard Hat
( ) Laser Eyewear

Gloves
(x) Cotton (Inner)
() PVC
() Leather
( ) Latex (Inner)
() Welding Gloves
(x) Nitrile
() Neoprene
() Insulating
() Vinyl
() Rubber (Outer)
() Other

Footwear
() Chemical Overboots
() Shoe Covers
(x) Other: Black rubber
(x) Steel-toed Leather
() Steel-toed Rubber
() Other

Health \& Safety Monitoring Requirements: No routine monitoring is anticipated during the sampling activities due to open air conditions. Initially, OVM measurements will be performed on samples as a precaution.

\section{Additional Comments / Changes}

1. Chemical hazards listed were found in small quantities in the sediments of the impoundments, and should pose no risk to the workers.

2. Heat and cold stress concerns will be addressed and emphasized at all times. All workers may use their option of stop work authority when dealing with heat and cold stress issues. Shaded rest areas and iced drinking fluids will be available at when heat stress is likely. A warm shelter will be available when necessary during cold weather.

3. In case of injuries or an emergency, workers should contact appropriate organizations such as the LSS office, etc. The emergency contacts list is available in the original Health \& Safety Plan and will be readily accessible at the work site. Evacuation procedures for general plant announcements will be followed.

4. When electrical equipment (pumps, lighting, etc.) is utilized at the site, GFCI outlets will be made available and used by workers. 
5. Hoisting and rigging plan is not required due to general lift activities. No critical lifts are anticipated. If a need arises, a lift plan will be developed and implemented.

6. If elevated work is required, the task will be performed under standard OSHA quidelines and regulations. This may involve the use of harness equipment and additional fall protection. The elevated work, if required, will be evaluated and discussed prior to beginning task, and any necessary controls will be implemented.

7. A site map is attached showing the approximate locations of zones for contamination control.

\section{Approvals}

(Original signed and located in project files)

$\overline{\text { Project Manager } \quad \text { Date }}$

$\overline{\text { Health \& Safety Officer } \quad \text { Date }}$

$\overline{\text { Health Physics (If Applicable) Date }}$

$\overline{\text { SHEST (If Applicable) } \quad \text { Date }}$

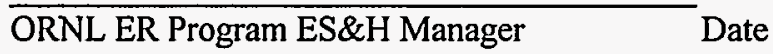

$\overline{\text { Other (Specify) Date }}$

$\overline{\text { Other (Specify) } \quad \text { Date }}$




\section{Site Layout \\ Ponds 3539 and 3540 Sampling Project}

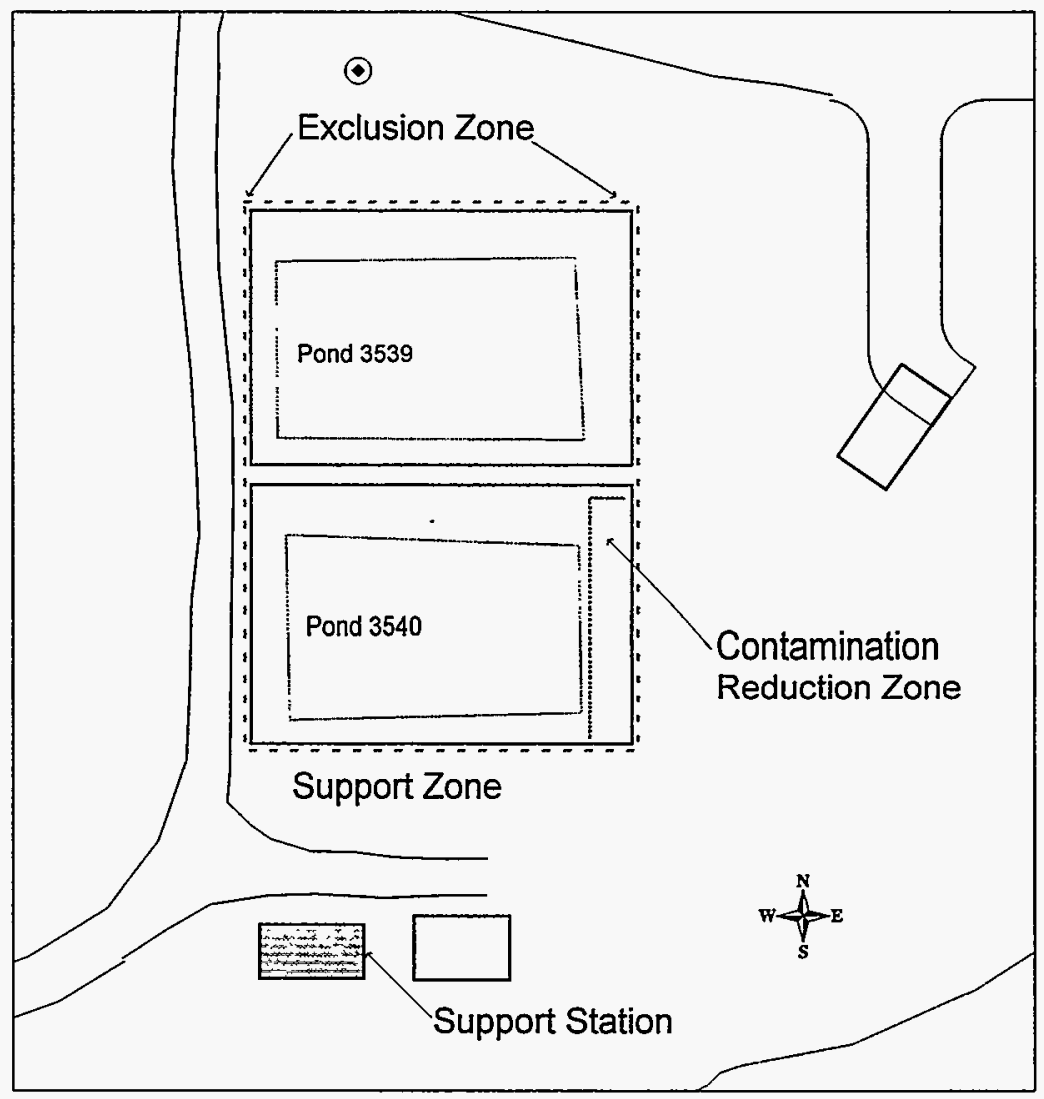




\section{INTERNAL DISTRIBUTION}

1. K. J. Brown

2. W. D. Brickeen

3-5. R. H. Coe III

6-8. R. A. Dean

9. R. D. Foley

10. R. C. Gosslee

11. C. A. Johnson

12-21. M. E. Murray

22-23. D. A. Rose
24. R. E. Swaja

25. M. S. Uziel

26. J. K. Williams

27. W. Winton

28. Central Research Library

29. ORNL Technical Library, $\mathrm{Y}-12$

30-32. MAD Records Center

33. Laboratory Records - RC

\section{EXTERNAL DISTRIBUTION}

34. P. T. Owen, Remedial Action Program Information Center, 138 Mitchell Road, Oak Ridge, TN 378307918

35-39. C. W. Mansfield, Project Manager, Bechtel Jacobs, 55 Jefferson Building, Oak Ridge, TN 37830

40-45. James Kopotic, Project Manager, 55 Jefferson Building, U.S. Department of Energy, Oak Ridge, TN 37830

46-47. Office of Scientific and Technical Information, U.S. Department of Energy, P.O. Box 62, Oak Ridge, TN 37831 